\title{
Unitary Approximations in Fault Detection Filter Design
}

\author{
Dušan Krokavec, Anna Filasová, and Pavol Liščinský \\ Department of Cybernetics and Artificial Intelligence, Faculty of Electrical Engineering and Informatics, Technical University of Košice, \\ Letná 9, 04200 Košice, Slovakia \\ Correspondence should be addressed to Dušan Krokavec; dusan.krokavec@tuke.sk
}

Received 19 January 2016; Revised 17 March 2016; Accepted 28 March 2016

Academic Editor: Zehui Mao

Copyright ( 2016 Dušan Krokavec et al. This is an open access article distributed under the Creative Commons Attribution License, which permits unrestricted use, distribution, and reproduction in any medium, provided the original work is properly cited.

\begin{abstract}
The paper is concerned with the fault detection filter design requirements that relax the existing conditions reported in the previous literature by adapting the unitary system principle in approximation of fault detection filter transfer function matrix for continuoustime linear MIMO systems. Conditions for the existence of a unitary construction are presented under which the fault detection filter with a unitary transfer function can be designed to provide high residual signals sensitivity with respect to faults. Otherwise, reflecting the emplacement of singular values in unitary construction principle, an associated structure of linear matrix inequalities with built-in constraints is outlined to design the fault detection filter only with a Hurwitz transfer function. All proposed design conditions are verified by the numerical illustrative examples.
\end{abstract}

\section{Introduction}

A conventional control for complex systems may result in unsatisfactory performances in the event of system component malfunctions. In order to remedy these weaknesses, different approaches to control system design are developed to tolerate component malfunctions and to maintain acceptable performances of the system with faults. The proposed control structures are known as fault-tolerant control (FTC) systems and they force the ability to accommodate component failures. In that sense, research in FTC is subject of a wide range of publications reflecting faults effect on control structure reconfiguration $[1,2]$ and fault estimation $[3,4]$, as well as fault residuals generation, analysis, and evaluation $[5,6]$. The ideal approach would be to construct disturbance-decoupled residuals, with responsiveness and sensibility to the faults, as shown in [7].

To scale up accuracy of fault detection, it is eligible to craft residuals with high sensitivity to faults under robustness to disturbances. One of the options is the use of $H_{\infty} / H_{-}$ optimization principle [8-11]. The restriction of this method is mainly the necessity of existence of a full rank directfeed external gain matrix from faults to residuals [12], which limits them to be used only to residuals revealing actuator faults. One of the other methods, based on unitary system properties, is proposed in $[13,14]$, where optimization is realized inherently in the sense that if the singular values of a unitary system are assigned as the magnitude frequency response of a first-order transfer function then strictly $H_{\infty}$ norm is the maximum and $H_{-}$index is the minimum of the generalized gain value of the transfer function. It should be noted that such approximation does not always exist, the construction is not unique, and the proposed design algorithm, exploiting the Riccati equation based formulation, can be often infeasible. The remarks, which admitted more properties of $H_{\infty}$ norm and $H_{-}$index, as well as other comments found to be very valuable in designing the unitary systems, are presented in Section 2.

Reflecting the basic results in unitary approximation of a square system transfer function matrix $[13,15]$, the principle was applied in [16] to design a unitary construction of the fault detection filter transfer function matrix. This technique, like that based on the classical procedure [14], is applicable to multivariable systems if the matching conditions are satisfied, that is, the unobservable modes of the used unitary approximation of the fault transform function matrix are stable. If these conditions are not satisfied, a feedback configuration of the fault detection filter, which optimizes sensitivity between the fault input and the output residuals, does not exist. 
To the best of our knowledge, so far, no more results than those given above on application of unitary approximations in the fault detection filter design are available in the literature, probably because the standard progression may not have a solution. This motivates this study, reflecting the matrix parameters implying an unstable unitary construction of the fault detection filter, to reformulate the design conditions in such a way that the fault detection filter with a Hurwitz transfer function matrix can be designed. The result is an associated structure of linear matrix inequalities, reflecting the principle of singular values emplacement in unitary construction, the associated state, and output variable constraints as well as an additive stabilizing feedback in fault detection filter. In this sense the proposed method attempts to combine the unitary approximation principle with the singular value placement to obtain a simple but practical algorithm for designing suboptimal performances of the fault detection filter transfer function matrix, that is, for a particular combination of three matrix parameters designed by the proposed method to find a fault detection filter with acceptable sensitivity, which cannot be reduced below some limiting value merely by manipulating stability of the filter.

Searching for gain matrices of the fault detection filter state-space description and ensuring the unitary model gain matrix values for a stable fault detection filter, the proposed design task is with unitary conditions reaching approximately the theoretical limits for the prescribed set of singular values. To analyze stability of the observer-based fault detection filter, the proposed conditions use standard arguments and require to solve only LMIs with the built-in prescribed constraints depending on the system output and fault input matrix structures. Within unitary solutions, the results are similar with those obtained by the method proposed in [17], but with reducing the ranges of all problem variables as much as possible.

The paper is organized as follows. Placed immediately after Introduction, Section 2 presents the problem statement and Section 3 summarizes in the basic preliminaries the auxiliary lemmas on the issue of the design task. The enhanced structure of unitary fault detection filter transfer function matrix, as well as the sets of LMIs, reflecting the quadratic Lyapunov function to describe the filter stability, is theoretically explained in Section 4 and the structures of the fault detections filters are given in Section 5. Two examples are provided to demonstrate the proposed approach in Section 6 and, finally, Section 7 draws some concluding remarks.

Used notations are conventional so that $\mathbf{x}^{T}, \mathbf{X}^{T}$ denote transpose of the vector $\mathbf{x}$ and matrix $\mathbf{X}$, respectively, $\mathbf{X}=$ $\mathbf{X}^{T}>0$ means that $\mathbf{X}$ is a symmetric positive definite matrix, the symbol $\mathbf{I}_{n}$ marks the $n$th order unit matrix, $\rho(\mathbf{X})$ and $\operatorname{rank}(\mathbf{X})$ indicate the eigenvalue spectrum and rank of a square matrix $\mathbf{X}, \mathbf{Y}^{\perp}$ designates the orthogonal complement to a rank-deficient matrix $\mathbf{Y}, \sigma_{i}(\mathbf{Z})$ labels the $i$ th singular value of matrix $\mathbf{Z}, \mathbb{R}$ denotes the set of real numbers, and $\mathbb{R}^{n}, \mathbb{R}^{n \times r}$ refer to the set of all $n$ dimensional real vectors and $n \times r$ real matrices, respectively.

\section{The Problem Statement}

The systems under consideration are linear MIMO continuous-time dynamic systems represented as follows:

$$
\begin{aligned}
& \dot{\mathbf{q}}(t)=\mathbf{A q}(t)+\mathbf{B u}(t)+\mathbf{F} \mathbf{f}(t)+\mathbf{E d}(t), \\
& \mathbf{y}(t)=\mathbf{C q}(t),
\end{aligned}
$$

where $\mathbf{q}(t) \in \mathbb{R}^{n}, \mathbf{u}(t) \in \mathbb{R}^{r}$, and $\mathbf{y}(t) \in \mathbb{R}^{m}$ are vectors of the state, input, and output variables, respectively, $\mathbf{f}(t) \in \mathbb{R}^{p}$ is fault vector, and $\mathbf{d}(t) \in \mathbb{R}^{r_{d}}$ is vector of disturbance. The real matrices $\mathbf{A} \in \mathbb{R}^{n \times n}, \mathbf{B} \in \mathbb{R}^{n \times r}, \mathbf{C} \in \mathbb{R}^{m \times n}, \mathbf{F} \in \mathbb{R}^{n \times p}$, and $\mathbf{E} \in \mathbb{R}^{n \times r_{d}}$ are finite values, satisfying the rank conditions $\operatorname{rank}(\mathbf{F})=p, \operatorname{rank}(\mathbf{C})=m, p=m$, and $p<n$. Moreover, it is supposed that the matrix $\mathbf{V}=\mathbf{C F}$ is regular matrix such that $\mathbf{V} \in \mathbb{R}^{m \times m}$

Problem of the interest is a unitary representation of the fault detection filter for the system with the square transfer function matrix of unknown fault input and the residuals. Note that such construction of unitary systems to given linear system, with respect to the singular values of the system transfer function matrix, is not a unique task also for square linear systems $[13,15]$.

\section{Basic Preliminaries}

3.1. Linear Systems. If $\mathscr{H}$ and $\mathscr{E}$ are Krein spaces [18], a continuous linear transformation

$$
\left(\begin{array}{ll}
\text { A } & \text { B } \\
\text { C } & \text { D }
\end{array}\right): \begin{array}{lll}
\mathscr{H} & & \mathscr{H} \\
\mathscr{E} & \oplus & \rightarrow \\
\mathscr{E}
\end{array}
$$

is called the linear system. The underlying Krein space $\mathscr{H}$ is called the state space and the auxiliary Krein space $\mathscr{E}$ is called the coefficient space or the external space [19]. The transformation $\mathbf{A}, \mathbf{B}, \mathbf{C}$ is the main input and output transformation, respectively, and the operator $\mathbf{D}$ is called the external operator. by

The transfer function $\mathbf{G}(s)$ of the linear system is defined

$$
\mathbf{G}(s)=\mathbf{C}\left(s \mathbf{I}_{n}-\mathbf{A}\right)^{-1} \mathbf{B}+\mathbf{D},
$$

where $\mathbf{A} \in \mathbb{R}^{n \times n}, \mathbf{B} \in \mathbb{R}^{n \times r}, \mathbf{C} \in \mathbb{R}^{m \times n}$, and $\mathbf{D} \in \mathbb{R}^{m \times r}$ are real matrices, $\mathbf{I}_{n} \in \mathbb{R}^{n \times n}$ is the identity matrix, and a complex number $s$ is the transform variable (Laplace variable) of the Laplace transform [20].

The eigenvalues of $\mathbf{A}$ are typically either real or complexconjugate pairs. If $\mathbf{A}$ has no imaginary eigenvalues then $\mathbf{G}(j \omega)$ is defined for all $\omega \in \mathbb{R}$, where $\omega$ is the frequency variable and $j:=\sqrt{-1}$. The singular values of the transfer function matrix $\mathbf{G}(s)$, evaluated on the imaginary axis, are $\sigma_{i}(\mathbf{G}(j \omega))$, where the $i$ th singular value of the complex matrix $\mathbf{G}(j \omega)$ is the nonnegative square-root of the $i$ th largest eigenvalue of $\mathbf{G}(j \omega) \mathbf{G}^{*}(j \omega)$, where $\mathbf{G}^{*}(j \omega)$ is the adjoint of $\mathbf{G}(j \omega)$. It is usually assumed that the singular values are ordered such that $\sigma_{i} \geq \sigma_{i+1}, i=1,2, \ldots, n-1$. 
Expressing the generalized gain of the system transfer function matrix $\mathbf{G}(s)$ as the 2-norm ratio of the input and output vectors, then the maximum and minimum singular values of $\mathbf{G}(j \omega)$ will constitute upper and lower bounds on this gain. The ratio between the maximum and minimum singular value is denoted by the condition number $\kappa$; that is,

$$
\kappa=\frac{\sigma_{\max }}{\sigma_{\min }}=\frac{\sigma_{1}}{\sigma_{n}} .
$$

The condition number plotted versus frequency variable $\omega$ outlines the system sensitivity to the direction of the input vector. If $\kappa \gg 1$, the generalized gain of the transfer function matrix will vary considerably with the input vector direction and $\mathbf{G}(s)$ is said to be ill-conditioned. Conversely, if $\kappa \approx 1$, the generalized gain of the transfer function matrix will be insensitive to the input direction and the system is said to be well-conditioned [21].

The $H_{\infty}$ norm of the transfer function matrix $\mathbf{G}(s)$ is [22]

$$
\|\mathbf{G}\|_{\infty}=\sup _{\omega \in \mathbb{R}} \sigma_{1}(\mathbf{G}(j \omega)) .
$$

The $H_{\infty}$ norm expresses the maximum of generalized gain of the system transfer function matrix for a class of input signals characterized by their 2-norm [23].

The $H_{-}$index of the transfer function matrix $\mathbf{G}(s)$ is defined as [8]

$$
\|\mathbf{G}\|_{-}=\inf _{\omega \in \mathbb{R}} \sigma_{n}(\mathbf{G}(j \omega)) .
$$

The $H_{-}$index expresses the minimum of generalized gain of the system transfer function matrix for a class of input signals characterized by their 2 -norm. Note that $H_{-}$index of a nonsquare system transfer function matrix is associated with rank of this matrix, that is, that $H_{-}$index is not completely dual to $H_{\infty}$ norm $[24,25]$.

It is evident that using the singular values, a system is assessed in more detail. Some other reflections can be found, for example, in [26-29].

Definition 1. A stable linear time-invariant system of $m$ inputs and $m$-outputs (square system) is defined as a unitary system if the singular values of its transfer function (transfer function matrix) $\mathbf{G}(s)$ satisfy [13]

$$
\sigma_{1}=\sigma_{2}=\cdots=\sigma_{m}
$$

where $\sigma_{i}$ is the $i$ th singular value of $\mathbf{G}(s)$.

Definition 2 (see [30]). Let $\mathbf{F} \in \mathbb{R}^{h \times h}, \operatorname{rank}(\mathbf{F})=k<h$ be a rank-deficient matrix. Then the null space $\mathbf{U}_{F}^{T}$ of $\mathbf{F}$ is the orthogonal complement of the row space of $\mathbf{F}$. An orthogonal complement $\mathbf{F}^{\perp}$ of $\mathbf{F}$ is

$$
\mathbf{F}^{\perp}=\mathbf{F}^{\circ} \mathbf{U}_{E}^{T},
$$

where $\mathbf{F}^{\circ}$ is an arbitrary matrix of appropriate dimension.

Considering the regular matrix $\mathbf{V}=\mathbf{C F}$, the following state coordinate transformation of system (1) can be done.
Lemma 3. If there are requirements for a regular matrix $\mathbf{V}$, defined as product of the matrix parameters $\mathbf{C}$ and $\mathbf{F}$ of the square system (1), to apply

$$
\begin{aligned}
\mathbf{C} & =\left[\begin{array}{ll}
\mathbf{V} & \mathbf{0}
\end{array}\right] \mathbf{T}, \\
\mathbf{T F} & =\left[\begin{array}{c}
\mathbf{I}_{m} \\
\mathbf{0}
\end{array}\right], \\
\mathbf{V} & =\mathbf{C F},
\end{aligned}
$$

then the transform matrix $\mathbf{T} \in \mathbb{R}^{n \times n}$ takes the form

$$
\mathbf{T}=\left[\begin{array}{c}
\mathbf{V}^{-1} \mathbf{C} \\
\mathbf{F}^{\perp}
\end{array}\right]
$$

where $m=p, \mathbf{V}^{-1} \mathbf{C} \in \mathbb{R}^{m \times n}$, and $\mathbf{F}^{\perp} \in \mathbb{R}^{(n-m) \times n}$, respectively, and $\mathbf{F}^{\perp}$ is the left orthogonal complement to $\mathbf{F}$.

Proof. Rewriting the first term of (9) as

$$
\mathbf{C}=\left[\begin{array}{ll}
\mathbf{V} & \mathbf{0}
\end{array}\right] \mathbf{T}=\left[\begin{array}{ll}
\mathbf{V} & \mathbf{0}
\end{array}\right]\left[\begin{array}{c}
\mathbf{T}_{1} \\
\mathbf{T}_{2}
\end{array}\right]=\mathbf{C},
$$

it is evident that

$$
\mathbf{V T}_{1}=\mathbf{C}, \quad \mathbf{T}_{1}=\mathbf{V}^{-1} \mathbf{C} .
$$

Analyzing the second term of (9), that is,

$$
\mathbf{T F}=\left[\begin{array}{c}
\mathbf{T}_{1} \\
\mathbf{T}_{2}
\end{array}\right] \mathbf{F}=\left[\begin{array}{c}
\mathbf{I}_{m} \\
\mathbf{0}
\end{array}\right],
$$

the following condition results:

$$
\mathbf{T}_{2} \mathbf{F}=\mathbf{0}, \quad \mathbf{T}_{2}=\mathbf{F}^{\perp}
$$

Thus, (12) and (14) imply (10).

It is easily verified using (11) and (14) that

$$
\begin{aligned}
\mathbf{T}_{1} \mathbf{F} & =\mathbf{V}^{-1} \mathbf{C F}=\mathbf{V}^{-1} \mathbf{V}=\mathbf{I}_{p}, \\
\mathbf{C T}^{-1} & =\left[\begin{array}{ll}
\mathbf{V} & \mathbf{0}
\end{array}\right],
\end{aligned}
$$

respectively. This concludes the proof.

3.2. Structures of Unitary Fault Transfer Function Matrices. The basic structure of the unitary fault transfer function matrix is introduced by the following lemma.

Lemma 4. For system (1) with $m=p$, the transform matrix T of the structure (10), and a prescribed positive scalar $s_{o} \in \mathbb{R}$ there exists the matrix $\mathbf{L}^{o} \in \mathbb{R}^{n \times m}$ such that the fault transfer function matrix can be approximated as

$$
\mathbf{G}_{f}(s) \approx \mathbf{G}_{\Delta}(s) \mathbf{G}_{f \Delta}(s),
$$


where

$$
\begin{aligned}
\mathbf{G}_{f \Delta}(s) & =\mathbf{C}\left(s \mathbf{I}_{n}-\left(\mathbf{A}-\mathbf{L}^{o} \mathbf{C}\right)\right)^{-1}=\frac{\mathbf{V}}{s+s_{o}}, \\
\mathbf{G}_{\Delta}(s) & =\mathbf{I}_{m}+\mathbf{C}\left(s \mathbf{I}_{n}-\mathbf{A}\right)^{-1} \mathbf{M}, \\
\mathbf{A}_{o} & =\mathbf{T A T}^{-1}=\left[\begin{array}{ll}
\mathbf{A}_{o 11} & \mathbf{A}_{o 12} \\
\mathbf{A}_{o 21} & \mathbf{A}_{o 22}
\end{array}\right], \\
\mathbf{L}^{o} & =\left[\begin{array}{c}
s_{o} \mathbf{I}_{m}+\mathbf{A}_{o 11} \\
\mathbf{A}_{o 21}
\end{array}\right], \\
\mathbf{M} & =\mathbf{T}^{-1} \mathbf{L}^{o} .
\end{aligned}
$$

Proof (compare $[13,16])$. Since

$$
\mathbf{G}_{f}(s)=\mathbf{C}\left(s \mathbf{I}_{n}-\mathbf{A}\right)^{-1} \mathbf{F}
$$

is the fault transfer function matrix of dimension $m \times m$, then (22) can be rewritten by using (9), (16), and (20) as

$$
\begin{aligned}
\mathbf{G}_{f}(s) & =\mathbf{C T}^{-1} \mathbf{T}\left(s \mathbf{I}_{n}-\mathbf{A}\right)^{-1} \mathbf{T}^{-1} \mathbf{T F} \\
& =\mathbf{C T}^{-1}\left(\mathbf{T}\left(s \mathbf{I}_{n}-\mathbf{A}\right) \mathbf{T}^{-1}\right)^{-1} \mathbf{T F} \\
& =\left[\begin{array}{ll}
\mathbf{V} & 0
\end{array}\right]\left(s \mathbf{I}_{n}-\mathbf{A}_{o}\right)^{-1}\left[\begin{array}{c}
\mathbf{I}_{p} \\
\mathbf{0}
\end{array}\right] .
\end{aligned}
$$

Specifying the matrix product $\mathbf{A}^{o}=\mathbf{T M C T}^{-1}$, where $\mathbf{M} \epsilon$ $\mathbb{R}^{n \times m}$ is a real matrix then, by exploiting (10) and (16), it yields

$$
\begin{aligned}
\mathbf{A}^{o} & =\mathbf{T M C T}^{-1}=\left[\begin{array}{c}
\mathbf{V}^{-1} \mathbf{C} \\
\mathbf{F}^{\perp}
\end{array}\right] \mathbf{M}\left[\begin{array}{ll}
\mathbf{V} & 0
\end{array}\right] \\
& =\left[\begin{array}{cc}
\mathbf{V}^{-1} \mathbf{C M V} & 0 \\
\mathbf{F}^{\perp} \mathbf{M V} & \mathbf{0}
\end{array}\right]
\end{aligned}
$$

and accepting the block matrix structure of (21) and (24), it can define

$$
\Delta \mathbf{A}_{o}=\mathbf{A}_{o}-\mathbf{A}^{o}=\left[\begin{array}{cc}
\mathbf{A}_{o 11}-\mathbf{V}^{-1} \mathbf{C M V} & \mathbf{A}_{o 12} \\
\mathbf{A}_{o 21}-\mathbf{F}^{\perp} \mathbf{M V} & \mathbf{A}_{o 22}
\end{array}\right] .
$$

Setting

$$
\begin{aligned}
\mathbf{A}_{o 11}-\mathbf{V}^{-1} \mathbf{C M V} & =-s_{o} \mathbf{I}_{m}, \\
\mathbf{A}_{o 21}-\mathbf{F}^{\perp} \mathbf{M V} & =\mathbf{0},
\end{aligned}
$$

where $s_{o} \in \mathbb{R}$ is a prescribed positive real value, and rewriting (26) as

$$
\left[\begin{array}{c}
s_{o} \mathbf{I}_{m}+\mathbf{A}_{o 11} \\
\mathbf{A}_{o 21}
\end{array}\right]=\left[\begin{array}{c}
\mathbf{V}^{-1} \mathbf{C} \\
\mathbf{F}^{\perp}
\end{array}\right] \mathbf{M V}=\mathbf{T M V}
$$

then, with

$$
\mathbf{M}=\mathbf{T}^{-1} \mathbf{L}^{o}
$$

it is

$$
\left[\begin{array}{c}
s_{o} \mathbf{I}_{m}+\mathbf{A}_{o 11} \\
\mathbf{A}_{o 21}
\end{array}\right]=\mathbf{T T}^{-1} \mathbf{L}^{o} \mathbf{V}=\mathbf{L}^{o} \mathbf{V} .
$$

Moreover, for (24) and (25), the following yields

$$
\begin{aligned}
\mathbf{A}^{o} & =\mathbf{T M C T}^{-1}=\mathbf{L}^{o} \mathbf{C T}^{-1}, \\
\Delta \mathbf{A}_{o} & =\mathbf{A}_{o}-\mathbf{A}^{o}=\mathbf{T}(\mathbf{A}-\mathbf{M C}) \mathbf{T}^{-1}=\mathbf{T} \Delta \mathbf{A T}^{-1},
\end{aligned}
$$

where

$$
\Delta \mathbf{A}=\mathbf{A}-\mathbf{M C}=\mathbf{A}-\mathbf{T}^{-1} \mathbf{L}^{o} \mathbf{C},
$$

and (25) takes the form

$$
\Delta \mathbf{A}_{o}=\left[\begin{array}{cc}
-s_{o} \mathbf{I}_{m} & \mathbf{A}_{o 12} \\
\mathbf{0} & \mathbf{A}_{o 22}
\end{array}\right] .
$$

Defining the transfer function matrix $\mathbf{G}_{f \Delta}(s)$ as

$$
\mathbf{G}_{f \Delta}(s)=\mathbf{C}\left(s \mathbf{I}_{n}-\Delta \mathbf{A}\right)^{-1} \mathbf{F},
$$

then with (32) it is

$$
\begin{aligned}
\mathbf{G}_{f \Delta}(s) & =\mathbf{C T}^{-1}\left(s \mathbf{I}_{n}-\mathbf{T} \Delta \mathbf{A} \mathbf{T}^{-1}\right)^{-1} \mathbf{T F} \\
& =\left[\begin{array}{ll}
\mathbf{V} & 0
\end{array}\right]\left(s \mathbf{I}_{n}-\Delta \mathbf{A}_{o}\right)^{-1}\left[\begin{array}{c}
\mathbf{I}_{p} \\
\mathbf{0}
\end{array}\right] .
\end{aligned}
$$

Since

$$
\begin{aligned}
& s \mathbf{I}_{n}-\Delta \mathbf{A}_{o}=\left[\begin{array}{cc}
\left(s+s_{o}\right) \mathbf{I}_{m} & -\mathbf{A}_{o 12} \\
\mathbf{0} & s \mathbf{I}_{n-m}-\mathbf{A}_{o 22}
\end{array}\right], \\
& \left(s \mathbf{I}_{n}-\Delta \mathbf{A}_{o}\right)^{-1} \\
& =\left[\begin{array}{cc}
\left(s+s_{o}\right)^{-1} \mathbf{I}_{m} & \left(s+s_{o}\right)^{-1} \mathbf{A}_{o 12}\left(s \mathbf{I}_{n-m}-\mathbf{A}_{o 22}\right)^{-1} \\
\mathbf{0} & \left(s \mathbf{I}_{n-m}-\mathbf{A}_{o 22}\right)^{-1}
\end{array}\right],
\end{aligned}
$$

substituting (36) into (34) it can obtain

$$
\mathbf{G}_{f \Delta}(s)=\left[\begin{array}{ll}
\mathbf{V} & \mathbf{0}
\end{array}\right]\left(s \mathbf{I}_{n}-\Delta \mathbf{A}_{o}\right)^{-1}\left[\begin{array}{c}
\mathbf{I}_{p} \\
\mathbf{0}
\end{array}\right]=\frac{\mathbf{V}}{s+s_{o}},
$$

which implies (18).

The transfer function (33) together with (31) and (22) can be written the way that

$$
\begin{aligned}
\mathbf{G}_{f \Delta}(s) & =\mathbf{C}\left(s \mathbf{I}_{n}-\Delta \mathbf{A}\right)^{-1} \mathbf{F} \\
& =\mathbf{C}\left(s \mathbf{I}_{n}-(\mathbf{A}-\mathbf{M C})\right)^{-1} \mathbf{F} \\
& =\mathbf{C}\left(\left(s \mathbf{I}_{n}-\mathbf{A}\right)\left(\mathbf{I}_{n}+\left(s \mathbf{I}_{n}-\mathbf{A}\right)^{-1} \mathbf{M C}\right)\right)^{-1} \mathbf{F} \\
& =\mathbf{C}\left(\mathbf{I}_{n}+\left(s \mathbf{I}_{n}-\mathbf{A}\right)^{-1} \mathbf{M C}\right)^{-1}\left(s \mathbf{I}_{n}-\mathbf{A}\right)^{-1} \mathbf{F}
\end{aligned}
$$


which gives, by using the equality

$$
(\mathrm{FC})^{-1} \mathrm{FC}=\mathbf{I}_{n}
$$

the expression for $\mathbf{G}_{f \Delta}(s)$ as follows:

$$
\begin{aligned}
& \mathbf{G}_{f \Delta}(s)=\mathbf{C}\left(\mathbf{I}_{n}+\left(s \mathbf{I}_{n}-\mathbf{A}\right)^{-1} \mathbf{M C}\right)^{-1}(\mathbf{F C})^{-1} \\
& \cdot \mathbf{F C}\left(s \mathbf{I}_{n}-\mathbf{A}\right)^{-1} \mathbf{F}=\mathbf{C}\left(\mathbf{I}_{n}+\left(s \mathbf{I}_{n}-\mathbf{A}\right)^{-1} \mathbf{M C}\right)^{-1} \\
& \cdot(\mathbf{F C})^{-1} \mathbf{F G}_{f}(s),
\end{aligned}
$$

where $\mathbf{G}_{f}(s)$, introduced in (22), is the fault transfer function matrix.

After some manipulations,

$$
\begin{aligned}
\mathbf{G}_{f \Delta}(s) & =\mathbf{C}\left((\mathbf{F C})\left(\mathbf{I}_{n}+\left(s \mathbf{I}_{n}-\mathbf{A}\right)^{-1} \mathbf{M C}\right)\right)^{-1} \mathbf{F G}_{f}(s) \\
& =\mathbf{C}\left(\mathbf{F}\left(\mathbf{I}_{m}+\mathbf{C}\left(s \mathbf{I}_{n}-\mathbf{A}\right)^{-1} \mathbf{M}\right) \mathbf{C}\right)^{-1} \mathbf{F G}_{f}(s),
\end{aligned}
$$

and $\mathbf{G}_{f \Delta}(s)$ can be approximated as

$$
\mathbf{G}_{f \Delta}(s) \approx\left(\mathbf{I}_{m}+\mathbf{C}\left(s \mathbf{I}_{n}-\mathbf{A}\right)^{-1} \mathbf{M}\right)^{-1} \mathbf{G}_{f}(s) .
$$

Thus, using (19) and then (41) implies (17). This concludes the proof.

With existence of such transformation, the structure of (24) really means that there exist the subset of transformed state variables whose dynamics is explicitly affected by the fault $\mathbf{f}(t)$ and a second one, whose dynamics is not affected explicitly by the fault $\mathbf{f}(t)$.

Remark 5. It is important to note the fact that the eigenvalues of $\mathbf{A}$ and of $\mathbf{A}_{o}$ are the same whenever $\mathbf{A}_{o}$ is related to $\mathbf{A}$ as $\mathbf{A}_{o}=\mathbf{T A T}^{-1}$ for any invertible T, as it is defined in (20) [31]. But this does not mean that if eigenvalues of the matrix $\mathbf{A}_{o}$ are stable then eigenvalues of the matrix $\mathbf{A}_{\text {o22 }}$ are also stable. This is a limitation of the methodology based on (32) and for a stable system it can lead to an unstable structure (35). It requires an additional stabilization, but this stabilization generally violates the desired unitary form of the fault transfer function matrix.

Defined by (7), a linear time-invariant system is considered as unitary if all singular values of its transfer function matrix are equal. Because the construction given in Lemma 4 is not unique, some equivalent structures can be used. One is introduced by the following lemma.

Lemma 6. An equivalent structure of the fault transfer function matrix of system (1) takes the form

$$
\mathbf{G}_{f}(s) \approx \mathbf{G}_{\Delta}^{\circ}(s)\left(\mathbf{G}_{f \Delta}^{\circ}(s)-\mathbf{V}\right),
$$

where, for a matrix $\mathbf{N} \in \mathbb{R}^{n \times m}$,

$$
\begin{aligned}
\mathbf{G}_{f \Delta}^{\circ}(s) & =\mathbf{C}\left(s \mathbf{I}_{n}-(\mathbf{A}-\mathbf{N C})\right)^{-1}(\mathbf{F}+\mathbf{N V})+\mathbf{V}, \\
\mathbf{G}_{\Delta}^{\circ}(s) & =\mathbf{I}_{m}+\mathbf{C}\left(s \mathbf{I}_{n}-\mathbf{A}\right)^{-1} \mathbf{N}
\end{aligned}
$$

Proof (compare [15]). Considering the associated system (44), it can be written for the resolvent matrix of a matrix $(\mathbf{A}-\mathbf{N C})$ that

$$
\begin{aligned}
& \left(s \mathbf{I}_{n}-(\mathbf{A}-\mathbf{N C})\right)^{-1} \\
& \quad=\left(\left(s \mathbf{I}_{n}-\mathbf{A}\right)\left(\mathbf{I}_{n}+\left(s \mathbf{I}_{n}-\mathbf{A}\right)^{-1} \mathbf{N C}\right)\right)^{-1} \\
& \quad=\mathbf{G}_{\Delta}^{\diamond}(s)\left(s \mathbf{I}_{n}-\mathbf{A}\right)^{-1}
\end{aligned}
$$

where

$$
\mathbf{G}_{\Delta}^{\diamond}(s)=\left(\mathbf{I}_{n}+\left(s \mathbf{I}_{n}-\mathbf{A}\right)^{-1} \mathbf{N C}\right)^{-1} .
$$

Therefore, the substitution of (9) and (46) in (44) leads to

$$
\begin{aligned}
\mathbf{G}_{f \Delta}^{\circ}(s) & =\mathbf{C G}_{\Delta}^{\diamond}(s)\left(s \mathbf{I}_{n}-\mathbf{A}\right)^{-1}(\mathbf{F}+\mathbf{N V})+\mathbf{V} \\
& =\mathbf{C}\left(\mathbf{G}_{\Delta}^{\diamond}(s)\left(s \mathbf{I}_{n}-\mathbf{A}\right)^{-1}\left(\mathbf{I}_{n}+\mathbf{N C}\right)+\mathbf{I}_{n}\right) \mathbf{F},
\end{aligned}
$$

and it yields with (47)

$$
\begin{aligned}
& \mathbf{G}_{f \Delta}^{\circ}(s) \\
& =\mathbf{C}\left(\mathbf{G}_{\Delta}^{\diamond}(s)\left(\left(s \mathbf{I}_{n}-\mathbf{A}\right)^{-1}+\left(s \mathbf{I}_{n}-\mathbf{A}\right)^{-1} \mathbf{N C}\right)+\mathbf{I}_{n}\right) \mathbf{F} \\
& =\mathbf{C}\left(\mathbf{G}_{\Delta}^{\diamond}(s)\left(\left(s \mathbf{I}_{n}-\mathbf{A}\right)^{-1}+\mathbf{G}_{\Delta}^{\diamond-1}(s)\right)\right) \mathbf{F} \\
& =\mathbf{C G}_{\Delta}^{\diamond}(s)\left(s \mathbf{I}_{n}-\mathbf{A}\right)^{-1} \mathbf{F}+\mathbf{C F} .
\end{aligned}
$$

Since using equality (39), it can obtain

$$
\begin{aligned}
\mathbf{C G}_{\Delta}^{\diamond}(s) & =\mathbf{C G}_{\Delta}^{\diamond}(s)(\mathbf{F C})^{-1} \mathbf{F C} \\
& =\mathbf{C}\left(\mathbf{F C}\left(\mathbf{I}_{n}+\left(s \mathbf{I}_{n}-\mathbf{A}\right)^{-1} \mathbf{N C}\right)\right)^{-1} \mathbf{F C} \\
& =\mathbf{C}\left(\mathbf{F}\left(\mathbf{I}_{m}+\mathbf{C}\left(s \mathbf{I}_{n}-\mathbf{A}\right)^{-1} \mathbf{N}\right) \mathbf{C}\right)^{-1} \mathbf{F C}
\end{aligned}
$$

and $\mathbf{C G}_{\Delta}^{\diamond}(s)$ can be approximated as

$$
\mathbf{C G}_{\Delta}^{\diamond}(s) \approx\left(\mathbf{I}_{m}+\mathbf{C}\left(s \mathbf{I}_{n}-\mathbf{A}\right)^{-1} \mathbf{N}\right)^{-1} \mathbf{C}=\mathbf{G}_{\Delta}^{\circ-1}(s) \mathbf{C},
$$

and then the substitution of (51) into (49) gives

$$
\begin{aligned}
\mathbf{G}_{f \Delta}^{\circ}(s) & \approx \mathbf{G}_{\Delta}^{\circ-1}(s) \mathbf{C}\left(s \mathbf{I}_{n}-\mathbf{A}\right)^{-1} \mathbf{F}+\mathbf{C F} \\
& =\mathbf{G}_{\Delta}^{\circ-1}(s) \mathbf{G}_{f}(s)+\mathbf{V} .
\end{aligned}
$$

Thus, (52) implies (43). This concludes the proof.

Corollary 7. Considering that $\mathbf{M}=\mathbf{N}$, (19) and (45) imply $\mathbf{G}_{\Delta}^{\circ}(s)=\mathbf{G}_{\Delta}(s)$. Since (17) defines $\mathbf{G}_{f}(s)=\mathbf{G}_{\Delta}(s) \mathbf{G}_{f \Delta}(s)$, it has to be satisfied with respect to (43) that

$$
\mathbf{G}_{f}(s)=\mathbf{G}_{\Delta}(s)\left(\mathbf{G}_{f \Delta}^{\circ}(s)-\mathbf{V}\right)=\mathbf{G}_{\Delta}(s) \mathbf{G}_{f \Delta}(s),
$$

which gives, with (18),

$$
\begin{aligned}
\mathbf{G}_{f \Delta}^{\circ}(s) & =\mathbf{G}_{f \Delta}(s)+\mathbf{V}=\mathbf{V}\left(\frac{1}{s+s_{o}}+1\right) \\
& =\frac{\mathbf{V}}{s+s_{o}}\left(s+s_{o}+1\right) ;
\end{aligned}
$$


that is

$$
\frac{\mathbf{G}_{f \Delta}^{\circ}(s)}{s+s_{o}+1}=\frac{\mathbf{V}}{s+s_{o}}=\mathbf{G}_{f \Delta}(s) .
$$

This corollary gives the possibility to combine the results of Lemmas 4 and 6 in the design of unitary fault transfer function matrix by the way specified in the following section.

\section{State-Space Description of Enhanced Structures}

To exploit the properties of the structures presented above, the enhanced form of unitary fault transfer matrix is proposed in the form

$$
\mathbf{G}_{f}^{\circ}(s)=\mathbf{C}\left(s \mathbf{I}_{n}-\mathbf{A}+(\mathbf{M}-\mathbf{N}) \mathbf{C}\right)^{-1} \mathbf{V}
$$

where $\mathbf{M}$ is introduced in (20) and $\mathbf{N}$ is designed in such a way that $\mathbf{F}+\mathbf{N V}=\mathbf{0}$.

To formulate the stability condition of the unitary system, approximated by the equivalent transform function matrix (56), the following theorems are given.

Theorem 8. The state-space representation of the enhanced structure of transfer function matrix (56) in the form of a closed-loop system is

$$
\begin{aligned}
\dot{\mathbf{q}}^{\circ}(t) & =(\mathbf{A}-\mathbf{J C}) \mathbf{q}^{\circ}(t)+\mathbf{V f}(t), \\
\mathbf{y}^{\circ}(t) & =\mathbf{C} \mathbf{q}^{\circ}(t), \\
\mathbf{w}^{\circ}(t) & =\mathbf{F}^{T} \mathbf{q}^{\circ}(t),
\end{aligned}
$$

where $\mathbf{w}^{\circ}(t)$ is the performance evaluation signal,

$$
\mathbf{J}=\mathbf{M}-\mathbf{N}, \quad \mathbf{N}=-\mathbf{F V}^{-1}, \mathbf{M}=\mathbf{T}^{-1} \mathbf{L}^{o},
$$

and the system constraint is

$$
\mathbf{R}^{\circ}=\left[\begin{array}{cc}
\mathbf{0} & \mathbf{S} \\
\mathbf{S}^{T} & -\mathbf{R}
\end{array}\right]=\left[\begin{array}{cc}
\mathbf{0} & \mathbf{F V} \\
\mathbf{V F}^{T} & -\mathbf{V} \mathbf{V}^{T}
\end{array}\right] \geq 0
$$

Proof. Use the Laplace transform property [32]; then (57) with the zero state vector initial condition implies

$$
\begin{aligned}
& \left(s \mathbf{I}_{n}-\mathbf{A}+\mathbf{J C}\right) \widetilde{\mathbf{q}}^{\circ}(s)=\mathbf{V} \widetilde{\mathbf{f}}(s), \\
& \widetilde{\mathbf{q}}^{\circ}(s)=\left(s \mathbf{I}_{n}-\mathbf{A}+(\mathbf{M}-\mathbf{N}) \mathbf{C}\right)^{-1} \mathbf{V} \widetilde{\mathbf{f}}^{\circ}(s),
\end{aligned}
$$

respectively, and

$$
\widetilde{\mathbf{y}}^{\circ}(s)=\mathbf{C}\left(s \mathbf{I}_{n}-\mathbf{A}+(\mathbf{M}-\mathbf{N}) \mathbf{C}\right)^{-1} \mathbf{V} \widetilde{\mathbf{f}}^{\circ}(s),
$$

while (64) implies (56).

Considering that

$$
\mathbf{F}+\mathbf{N V}=\mathbf{0},
$$

premultiplying the right side of (65) by $\mathbf{V}^{T}$ leads to

$$
\mathbf{F V}^{T}+\mathbf{N V V} \mathbf{V}^{T}=\mathbf{0}
$$

which implies that

$$
\mathbf{N}=-\mathbf{F V}^{T}\left(\mathbf{V V}^{T}\right)^{-1}
$$

Since $\mathbf{V}$ is a regular matrix, postmultiplying the right side of (67) by $\mathbf{V F}^{T}$ gives

$$
\mathbf{F V}^{T}\left(\mathbf{V V}^{T}\right)^{-1} \mathbf{V} \mathbf{F}^{T}=\mathbf{F F}^{T}=-\mathbf{N V F}^{T} \geq 0
$$

Thus, (59) implies

$$
\begin{aligned}
\mathbf{w}^{\circ T}(t) \mathbf{w}^{\circ}(t) & =\mathbf{q}^{\circ T}(t) \mathbf{F}^{T} \mathbf{F} \mathbf{q}^{\circ}(t) \\
& =\mathbf{q}^{\circ T}(t) \mathbf{F} \mathbf{V}^{T}\left(\mathbf{V} \mathbf{V}^{T}\right)^{-1} \mathbf{V} \mathbf{F}^{T} \mathbf{q}^{\circ}(t) ;
\end{aligned}
$$

it is evident that (69) is the constraint given on $\mathbf{q}^{\circ}(t)$ and using the Schur complement property (68) implies quadratic constraint (61).

Finally, since V is regular, (65) implies (60). This concludes the proof.

Theorem 9. The equivalent system (57) and (58) is stable if there exists a symmetric positive definite matrix $\mathbf{P} \in \mathbb{R}^{n \times n}$ such that

$$
\begin{aligned}
& \mathbf{P}=\mathbf{P}^{T}>0, \\
& {\left[\begin{array}{cc}
\mathbf{P}(\mathbf{A}-\mathbf{J C})+(\mathbf{A}-\mathbf{J C})^{T} \mathbf{P} & \mathbf{F V} \\
\mathbf{V F}^{T} & -\mathbf{V} \mathbf{V}^{T}
\end{array}\right]<0, }
\end{aligned}
$$

and the common gain matrix is given in (60).

Proof. Since the Lyapunov function candidate can be considered in the form

$$
v\left(\mathbf{q}^{\circ}(t)\right)=\mathbf{q}^{\circ T}(t) \mathbf{P} \mathbf{q}^{\circ}(t)+\int_{0}^{t} \mathbf{w}^{\circ T}(v) \mathbf{w}^{\circ}(v) \mathrm{d} v>0,
$$

where $\mathbf{P} \in \mathbb{R}^{n \times n}$ is a symmetric, positive, and definite matrix, then the time derivative of (72) can be written as

$$
\begin{aligned}
\dot{v}\left(\mathbf{q}^{\circ}(t)\right)= & \dot{\mathbf{q}}^{\circ T}(t) \mathbf{P} \mathbf{q}^{\circ}(t)+\mathbf{q}^{\circ T}(t) \mathbf{P} \dot{\mathbf{q}}^{\circ}(t) \\
& +\mathbf{w}^{\circ T}(t) \mathbf{w}^{\circ}(t)<0
\end{aligned}
$$

Substituting (69), as well as (57) for the fault-free regime, into (73), then inequality (73) can be rewritten as

$$
\begin{aligned}
\dot{v}\left(\mathbf{q}^{\circ}(t)\right)= & \mathbf{q}^{\circ T}(t)(\mathbf{A}-\mathbf{J C})^{T} \mathbf{P} \mathbf{q}^{\circ}(t) \\
& +\mathbf{q}^{\circ T}(t) \mathbf{P}(\mathbf{A}-\mathbf{J C}) \mathbf{q}^{\circ}(t) \\
& +\mathbf{q}^{\circ T}(t) \mathbf{F} \mathbf{V}^{T}\left(\mathbf{V} \mathbf{V}^{T}\right)^{-1} \mathbf{V F}^{T} \mathbf{q}^{\circ}(t)<0
\end{aligned}
$$

which implies

$$
(\mathbf{A}-\mathbf{J C})^{T} \mathbf{P}+\mathbf{P}(\mathbf{A}-\mathbf{J C})+\mathbf{F V}^{T}\left(\mathbf{V V}^{T}\right)^{-1} \mathbf{V F} \mathbf{F}^{T}<0 .
$$

Thus, using the Schur complement, (75) implies (71). This concludes the proof. 
If the set of eigenvalues of $\mathbf{A}_{o 22}$ contains an unstable eigenvalue, the conditions have to be extended to design a stable fault detection filter (a fault detection filter with the Hurwitz transfer function [33]). Because the matrix block $\mathbf{A}_{o 22}$ is unstable and unobservable in the form of the statespace description (57), the synthesis of an additional observer gain is required to use a dual form, considering that the couple $(\mathbf{A}, \mathbf{C})$ is observable. Moreover, to obtain sufficient dynamic range of residual signals, in the solution could be included system output constraints.

Theorem 10. The equivalent system (57) and (58) with unstable matrix block $\mathbf{A}_{o 22}$ is stabilizable if there exists a symmetric positive definite matrix $\mathbf{Q} \in \mathbb{R}^{n \times n}$, a regular matrix $\mathbf{S} \in \mathbb{R}^{m \times m}$, and a matrix $\mathbf{U} \in \mathbb{R}^{n \times m}$ such that

$$
\begin{aligned}
& \mathbf{Q}=\mathbf{Q}^{T}>0, \\
& {\left[\begin{array}{cc}
\mathbf{Q}(\mathbf{A}-\mathbf{J C})^{T}+(\mathbf{A}-\mathbf{J C}) \mathbf{Q}-\mathbf{U C}-\mathbf{C}^{T} \mathbf{U}^{T} & \mathbf{Q} \mathbf{C}^{T}+\mathbf{F V}^{T} \\
\mathbf{C Q}+\mathbf{V F}^{T} & -\mathbf{V V}^{T}
\end{array}\right]} \\
& \quad<0, \\
& \mathbf{C Q}=\mathbf{S C},
\end{aligned}
$$

where the matrix $\mathbf{J}$ is given in (60).

If (76)-(78) are admissible, then

$$
\begin{aligned}
\mathbf{H} & =\mathbf{U S}^{-1}, \\
\mathbf{J}^{\circ} & =\mathbf{J}+\mathbf{H}, \\
\mathbf{G}_{f}^{\circ}(s) & =\mathbf{C}\left(s \mathbf{I}_{n}-\mathbf{A}+\mathbf{J}^{\circ} \mathbf{C}\right)^{-1} \mathbf{V} .
\end{aligned}
$$

Proof. Writing autonomous, fault-free free model (1) in the dual state-space form [34] as

$$
\begin{aligned}
\dot{\mathbf{p}}(t) & =\mathbf{A}^{T} \mathbf{p}(t)+\mathbf{C}^{T} \mathbf{d}(t), \\
\mathbf{y}(t) & =\mathbf{E}^{T} \mathbf{p}(t),
\end{aligned}
$$

and then considering the Lyapunov function candidate of the form

$$
v(\mathbf{p}(t))=\mathbf{p}^{T}(t) \mathbf{Q p}(t)>0,
$$

where $\mathbf{Q} \in \mathbb{R}^{n \times n}$ is a positive definite matrix, it has to be

$$
\dot{v}(\mathbf{p}(t))=\dot{\mathbf{p}}^{T}(t) \mathbf{Q p}(t)+\mathbf{p}^{T}(t) \mathbf{Q} \dot{\mathbf{p}}(t)<0,
$$

and by substituting (80) into (83) it can obtain

$$
\begin{aligned}
\dot{v}(\mathbf{p}(t))= & \left(\mathbf{A}^{T} \mathbf{p}(t)+\mathbf{C}^{T} \mathbf{d}(t)\right)^{T} \mathbf{Q p}(t) \\
& +\mathbf{p}^{T}(t) \mathbf{Q}\left(\mathbf{A}^{T} \mathbf{p}(t)+\mathbf{C}^{T} \mathbf{d}(t)\right)<0 .
\end{aligned}
$$

Introducing the notation

$$
\mathbf{p}^{\circ T}(t)=\left[\begin{array}{ll}
\mathbf{p}^{T}(t) & \mathbf{d}^{T}(t)
\end{array}\right]
$$

then (84) can be redefined using the Krasovskii theorem (see, e.g., [35]), as

$$
\dot{v}\left(\mathbf{p}^{\circ}(t)\right)=\mathbf{p}^{\circ T}(t) \mathbf{Q}^{\circ} \mathbf{p}^{\circ}(t) \leq-\mathbf{p}^{\circ T}(t) \mathbf{R}^{\circ} \mathbf{p}^{\circ}(t)<0,
$$

where $\mathbf{R}^{\circ}$ is given in (61). Then (86) implies

$$
\dot{v}\left(\mathbf{p}^{\circ}(t)\right)=\mathbf{p}^{\circ T}(t)\left(\mathbf{Q}^{\circ}+\mathbf{R}^{\circ}\right) \mathbf{p}^{\circ}(t)<0,
$$

where

$$
\mathbf{Q}^{\circ}+\mathbf{R}^{\circ}=\left[\begin{array}{cc}
\mathbf{Q A}^{T}+\mathbf{A Q} & \mathbf{Q C}^{T}+\mathbf{F V}^{T} \\
\mathbf{C Q}+\mathbf{V F}^{T} & -\mathbf{V V}^{T}
\end{array}\right]<0 .
$$

Inserting $\mathbf{A} \leftarrow(\mathbf{A}-\mathbf{J C}-\mathbf{H C})$, where $\mathbf{H} \in \mathbb{R}^{n \times n}$ is an additive observer gain then

$$
\begin{aligned}
& {\left[\begin{array}{cc}
\mathbf{Q}(\mathbf{A}-\mathbf{J C}-\mathbf{H C})^{T}+(\mathbf{A}-\mathbf{J C}-\mathbf{H C}) \mathbf{Q} \mathbf{Q C}^{T}+\mathbf{F V}^{T} \\
\mathbf{C Q}+\mathbf{V F}^{T} & -\mathbf{V V}^{T}
\end{array}\right]} \\
& \quad<0 .
\end{aligned}
$$

Setting

$$
\mathrm{HCQ}=\mathrm{HSS}^{-1} \mathrm{CQ}=\mathrm{UCQ}^{-1} \mathbf{Q}=\mathrm{UC},
$$

where $\mathbf{S} \in \mathbb{R}^{m \times m}$ is a regular matrix and

$$
\begin{aligned}
\mathbf{U} & =\mathbf{H S}, \\
\mathbf{S}^{-1} \mathbf{C} & =\mathrm{CQ}^{-1},
\end{aligned}
$$

then (89) implies (77) and (91) gives (78). This concludes the proof.

\section{Fault Detection Filters}

Denoting

$$
\begin{gathered}
\mathbf{e}(t)=\mathbf{q}^{\circ}(t), \\
\mathbf{J}=\mathbf{M}-\mathbf{N}, \\
(\mathbf{J}=\mathbf{M}-\mathbf{N}+\mathbf{H}),
\end{gathered}
$$

it yields in the fault-free regime

$$
\dot{\mathbf{e}}(t)=(\mathbf{A}-\mathbf{J C}) \mathbf{e}(t),
$$

which is the autonomous equation of the estimation error of Luenberger observer to the nominal noise and fault-free system (1), defined in the form [4]

$$
\dot{\mathbf{q}}_{e}(t)=\mathbf{A} \mathbf{q}_{e}(t)+\mathbf{B u}(t)+\mathbf{J C}\left(\mathbf{q}(t)-\mathbf{q}_{e}(t)\right) .
$$

Introducing $\mathbf{e}(t)=\mathbf{q}(t)-\mathbf{q}_{e}(t)$, then with (1) and (94) it yields

$$
\begin{aligned}
\dot{\mathbf{e}}(t)= & \mathbf{A q}(t)+\mathbf{B u}(t)+\mathbf{F f}(t)+\mathbf{E d}(t)-\mathbf{A} \mathbf{q}_{e}(t) \\
& -\mathbf{B u}(t)-\mathbf{J C}\left(\mathbf{q}(t)-\mathbf{q}_{e}(t)\right) \\
= & (\mathbf{A}-\mathbf{J C}) \mathbf{e}(t)+\mathbf{F f}(t)+\mathbf{E d}(t), \\
\widetilde{\mathbf{e}}(s)= & \left(s \mathbf{I}_{n}-(\mathbf{A}-\mathbf{J C})\right)^{-1}(\tilde{\mathbf{F}}(s)+\mathbf{E} \widetilde{\mathbf{d}}(s)),
\end{aligned}
$$


respectively, where $\widetilde{\mathbf{e}}(s), \widetilde{\mathbf{f}}(s)$, and $\widetilde{\mathbf{d}}(s)$ stand for the Laplace transform of the $n$ dimensional observer error vector, the $m$ dimensional fault vector, and the $p$ dimensional disturbance vector.

Designing the fault residuals as

$$
\begin{aligned}
\mathbf{r}(t) & =\mathbf{V}^{-1} \mathbf{C e}(t), \\
\widetilde{\mathbf{r}}(s) & =\mathbf{V}^{-1} \mathbf{C} \widetilde{\mathbf{e}}(s),
\end{aligned}
$$

then the fault detection filter transfer function matrices of the fault and the disturbance are

$$
\begin{aligned}
& \mathbf{G}_{f}(s)=\mathbf{V}^{-1} \mathbf{C}\left(s \mathbf{I}_{n}-(\mathbf{A}-\mathbf{J C})\right)^{-1} \mathbf{F}, \\
& \mathbf{G}_{d}(s)=\mathbf{V}^{-1} \mathbf{C}\left(s \mathbf{I}_{n}-(\mathbf{A}-\mathbf{J C})\right)^{-1} \mathbf{E} .
\end{aligned}
$$

It is evident that, with $\mathbf{J}$ of the structure $(60), \mathbf{G}_{f}(s)$ is a unitary transfer function matrix with optimized singular values related properties.

\section{Illustrative Examples}

To illustrate the design principles, two examples are presented. First, Theorems 9 and 10 are applied to study the unitary fault detection transfer function matrix design problems in Example 1. Then, considering a real world model of the chemical reactor [36], the directional residuals properties are demonstrated in Example 2.

Example 1. The linear system, represented by model (1), is with the matrix parameters

$$
\begin{aligned}
& \mathbf{A}=\left[\begin{array}{cccc}
-1.5780 & -3.1660 & -2.1392 & -1.1056 \\
1.5478 & -1.5604 & -1.5612 & -5.9870 \\
3.6630 & 2.5695 & -1.5962 & -5.6508 \\
0.0947 & 4.9043 & 6.7035 & -1.4961
\end{array}\right] \text {, } \\
& \mathbf{B}=\left[\begin{array}{cc}
0.0000 & -0.7674 \\
-1.5953 & -0.6395 \\
-1.5404 & -0.6380 \\
1.0513 & 0.8971
\end{array}\right] \text {, } \\
& \mathbf{F}=\left[\begin{array}{cc}
0.0000 & -0.7674 \\
-1.5953 & -0.6395 \\
\pm 1.5404 & -0.6380 \\
1.0513 & 0.8971
\end{array}\right] \text {, } \\
& \mathbf{E}=\left[\begin{array}{c}
-1.5780 \\
1.5478 \\
3.6630 \\
0.0947
\end{array}\right] \\
& C=\left[\begin{array}{cccc}
0.0000 & -1.8600 & 0.0000 & 1.9003 \\
0.4970 & 0.0000 & -1.8805 & 0.0000
\end{array}\right] \text {. }
\end{aligned}
$$

Considering the signum $(+)$ in the $(3,1)$ matrix element of $\mathbf{F}$ then

$$
\mathbf{V}=\mathbf{C F}=\left[\begin{array}{cc}
4.9650 & 2.8942 \\
-2.8967 & 0.8184
\end{array}\right], \quad \operatorname{det}(\mathbf{V}) \neq 0
$$

and the parameters of the matrix $\mathbf{T}$ are computed as follows:

$$
\begin{aligned}
\mathbf{V}^{-1} \mathbf{C} & =\left[\begin{array}{cccc}
-0.1156 & -0.1223 & 0.4373 & 0.1249 \\
0.1983 & -0.4329 & -0.7501 & 0.4422
\end{array}\right], \\
\mathbf{F}^{\perp} & =\left[\begin{array}{cccc}
-0.8366 & 0.2595 & 0.4248 & -0.2286 \\
0.1130 & 0.6631 & 0.2007 & 0.7122
\end{array}\right], \\
\mathbf{T} & =\left[\begin{array}{cccc}
-0.1156 & -0.1223 & 0.4373 & 0.1249 \\
0.1983 & -0.4329 & -0.7501 & 0.4422 \\
-0.8366 & 0.2595 & 0.4248 & -0.2286 \\
0.1130 & 0.6631 & 0.2007 & 0.7122
\end{array}\right] .
\end{aligned}
$$

Computing (20) and separating the blocks of the matrix $\mathbf{A}^{\circ}$ give the results

$$
\begin{aligned}
& \mathbf{A}_{o 11}=\left[\begin{array}{ll}
-4.6582 & -4.6807 \\
12.5921 & 5.1671
\end{array}\right], \\
& \mathbf{A}_{o 12}=\left[\begin{array}{ll}
-2.3248 & 0.4532 \\
4.4851 & 4.3760
\end{array}\right], \\
& \mathbf{A}_{o 21}=\left[\begin{array}{ll}
-7.6293 & -5.7984 \\
-5.8979 & -10.6135
\end{array}\right], \\
& \mathbf{A}_{o 22}=\left[\begin{array}{ll}
-4.2384 & -0.1943 \\
-4.0113 & -2.5012
\end{array}\right],
\end{aligned}
$$

where the stable eigenvalue spectrum

$$
\rho\left(\mathbf{A}_{o 22}\right)=\{-4.6082,-2.1314\}
$$

gives the possibility to obtain a unitary fault detection filter.

Thus, choosing $s_{o}=5$, it is obtained using (20) and (60) that

$$
\begin{aligned}
\mathbf{L}^{o} & =\left[\begin{array}{cc}
0.3418 & -4.6807 \\
12.5921 & 10.1671 \\
-7.6293 & -5.7984 \\
-5.8979 & -10.6135
\end{array}\right], \\
\mathbf{M} & =\left[\begin{array}{cc}
-0.0142 & -0.2290 \\
-2.7392 & 0.2023 \\
-3.0371 & -3.5498 \\
-0.6039 & -3.1706
\end{array}\right],
\end{aligned}
$$




$$
\begin{aligned}
\mathbf{N} & =\left[\begin{array}{cc}
0.1786 & 0.3061 \\
0.2537 & -0.1159 \\
0.0472 & 0.6127 \\
-0.2779 & -0.1134
\end{array}\right], \\
\mathbf{J} & =\left[\begin{array}{cc}
-0.1927 & -0.5352 \\
-2.9929 & 0.3181 \\
-3.0843 & -4.1625 \\
-0.3260 & -3.0572
\end{array}\right],
\end{aligned}
$$

respectively. Thus, constructing the system matrix

$$
\begin{aligned}
\mathbf{A}_{e} & =\mathbf{A}-\mathbf{J C} \\
& =\left[\begin{array}{cccc}
-1.3120 & -3.5245 & -3.1456 & -0.7393 \\
1.3897 & -7.1272 & -0.9629 & -0.2996 \\
5.7318 & -3.1673 & -9.4238 & 0.2103 \\
1.6141 & 4.2980 & 0.9545 & -0.8766
\end{array}\right],
\end{aligned}
$$

the stable eigenvalues spectrum of $\mathbf{A}_{e}$ is

$$
\rho\left(\mathbf{A}_{e}\right)=\left\{\begin{array}{llll}
-2.1314 & -4.6082 & -6.0000 & -6.0000
\end{array},,\right.
$$

and the steady-state value of the fault detection filter transfer function matrix is

$$
\mathbf{G}_{f}(0)=-\mathbf{V}^{-1} \mathbf{C A}_{e}^{-1} \mathbf{F}=\left[\begin{array}{ll}
0.1667 & 0.0000 \\
0.0000 & 0.1667
\end{array}\right] .
$$

For completeness it can be verified that in sense of Lyapunov stability there exists the positive definite matrix $\mathbf{P}$ such that (70) and (71) are affirmative.

Considering the sign (-) in $\mathbf{F}$, that is, $\mathbf{F}=\mathbf{B}$, it changes signum of the $(2,1)$ element of $\mathbf{V}$ that is

$$
\mathbf{V}=\mathbf{C F}=\left[\begin{array}{ll}
4.9650 & 2.8942 \\
2.8967 & 0.8184
\end{array}\right], \quad \operatorname{det}(\mathbf{V}) \neq 0,
$$

and the parameters of the matrix $\mathbf{T}$ are now computed as follows:

$$
\begin{aligned}
\mathbf{V}^{-1} \mathbf{C} & =\left[\begin{array}{cccc}
0.3329 & 0.3523 & -1.2597 & -0.3599 \\
-0.5711 & -1.2470 & 2.1610 & 1.2741
\end{array}\right], \\
\mathbf{F}^{\perp} & =\left[\begin{array}{cccc}
0.1753 & -0.5316 & 0.7661 & 0.3158 \\
0.4507 & 0.5187 & -0.0422 & 0.7253
\end{array}\right], \\
\mathbf{T} & =\left[\begin{array}{cccc}
0.3329 & 0.3523 & -1.2597 & -0.3599 \\
-0.5711 & -1.2470 & 2.1610 & 1.2741 \\
0.1753 & -0.5316 & 0.7661 & 0.3158 \\
0.4507 & 0.5187 & -0.0422 & 0.7253
\end{array}\right] .
\end{aligned}
$$

Computing (20) and separating the blocks of the matrix $\mathbf{A}^{\circ}$ give now the results

$$
\begin{aligned}
& \mathbf{A}_{o 11}=\left[\begin{array}{cc}
18.5471 & 13.4846 \\
-43.8674 & -25.9954
\end{array}\right], \\
& \mathbf{A}_{o 12}=\left[\begin{array}{ll}
-9.8798 & -3.0397 \\
12.5359 & 10.1353
\end{array}\right], \\
& \mathbf{A}_{o 21}=\left[\begin{array}{ll}
-10.0330 & -6.2445 \\
-11.4737 & -6.7856
\end{array}\right], \\
& \mathbf{A}_{o 22}=\left[\begin{array}{cc}
2.9984 & 2.1854 \\
4.2953 & -1.7808
\end{array}\right],
\end{aligned}
$$

where the unstable eigenvalue spectrum

$$
\rho\left(\mathbf{A}_{o 22}\right)=\{4.4943,-3.2767\}
$$

does not give the possibility to obtain a strictly unitary fault detection filter.

As above, choosing $s_{o}=5$, it is obtained using (20) and (60) that

$$
\begin{aligned}
\mathbf{L}^{o} & =\left[\begin{array}{ccc}
5.8844 & 4.1078 & -0.9991 \\
-11.1342 & 31.0383 & -8.0335 \\
8.6811 & -3.7656 & 10.7293 \\
-4.0432 & 19.2186 & -2.6660
\end{array}\right], \\
\mathbf{M} & =\left[\begin{array}{cc}
-1.5138 & 5.0746 \\
-3.4282 & 2.6391 \\
-4.9462 & 3.2020 \\
-0.1713 & -4.7003
\end{array}\right], \\
\mathbf{N} & =\left[\begin{array}{cc}
0.5145 & -0.8819 \\
0.1266 & 0.3338 \\
0.1360 & 0.2987 \\
-0.4023 & 0.3267
\end{array}\right], \\
\mathbf{J} & =\mathbf{M}-\mathbf{N}=\left[\begin{array}{cc}
-2.0283 & 5.9564 \\
-3.5548 & 2.3054 \\
-5.0822 & 2.9033 \\
0.2310 & -5.0270
\end{array}\right],
\end{aligned}
$$

which results in the following unstable eigenvalues spectrum

$$
\begin{aligned}
\rho\left(\mathbf{A}_{e}\right) & =\rho(\mathbf{A}-\mathbf{J C}) \\
& =\left\{\begin{array}{llll}
4.4943 & -3.2767 & -6.0000 & -6.0000
\end{array}\right\} .
\end{aligned}
$$

It is evident that using the coordinate transformation defined by the transform matrix (10) and the block matrix $\mathbf{A}_{o 22}$ in the matrix structure (20) is unobservable in the structure (57)-(58), while the eigenvalues of $\mathbf{A}_{o 22}$ determine the unprescribed subset of eigenvalues of $\rho\left(\mathbf{A}_{e}\right)$ (compare $\rho\left(\mathbf{A}_{e}\right)$ and $\rho \mathbf{A}_{o 22}$ in this part of example). 
To stabilize the fault detection filter, an additive gain $\mathbf{H} \epsilon$ $\mathbb{R}^{n \times m}$ is computed solving the set of inequalities (76) and (77) and equality (78), where, with

$$
\begin{aligned}
& \mathbf{U}=\left[\begin{array}{cc}
14.0196 & -42.1540 \\
26.1053 & 12.0055 \\
15.8767 & -10.3000 \\
2.2226 & -0.2937
\end{array}\right], \\
& \mathbf{S}=\left[\begin{array}{ll}
9.5763 & 5.0779 \\
2.7170 & 7.2266
\end{array}\right],
\end{aligned}
$$

it is obtained that

$$
\begin{aligned}
& \mathbf{H}=\left[\begin{array}{ll}
3.8956 & -8.5706 \\
2.8161 & -0.3175 \\
2.5758 & -3.2353 \\
0.3043 & -0.2545
\end{array}\right], \\
& \mathbf{J}^{\circ}=\mathbf{J}+\mathbf{H}=\left[\begin{array}{cc}
1.8673 & -2.6141 \\
-0.7387 & 1.9879 \\
-2.5064 & -0.3320 \\
0.5353 & -5.2815
\end{array}\right] .
\end{aligned}
$$

This ensures the stable eigenvalues spectrum of $\mathbf{A}_{e}$ as follows:

$$
\begin{aligned}
\rho\left(\mathbf{A}-\mathbf{J}^{\circ} \mathbf{C}\right) \\
\quad=\{-2.3882+6.4561 i-1.5853+4.9788 i\},
\end{aligned}
$$

but the fault detection filter transfer function matrix is not unitary and works with the steady-state value

$$
\mathbf{G}_{f}(0)=-\mathbf{V}^{-1} \mathbf{C A}_{e}^{-1} \mathbf{F}=\left[\begin{array}{cc}
-0.0484 & 0.0474 \\
0.2132 & 0.0262
\end{array}\right] \text {. }
$$

It is obvious that for a stable $\mathbf{A}_{o 22}$ the fault detection filter with a unitary transfer function can be designed, while for an unstable $\mathbf{A}_{\text {o22 }}$ the fault detection filter only with a Hurwitz transfer function can be nominated.

Because the matrix A is not Hurwitz, for the simulation purpose, the system is stabilized using the state feedback control law

$$
\mathbf{u}(t)=-\mathbf{K q}(t)
$$

where $\mathbf{K} \in \mathbb{R}^{3 \times 4}$ is the gain matrix. Since, according to the separation principle, the control gain matrix can be designed independently of the fault detection filter parameters design, the MATLAB function $K=$ place(.) is used. Designing, for simplicity, with the prescribed desired set of closed-loop system matrix eigenvalues $\rho(\mathbf{A}-\mathbf{B K})=\{-1,-2,-3,-4\}$, the gain matrix is

$$
\mathbf{K}=\left[\begin{array}{cccc}
-0.8205 & -1.7778 & -0.1700 & 2.6615 \\
-1.1104 & 3.5495 & 3.0193 & 1.3570
\end{array}\right]
$$

Note the control law design could be created, for example, using the bounded real lemma LMI to reflect the $H_{\infty}$ norm of the disturbance transfer function matrix (see, e.g., [37]), though this still does not solve completely the problem of integrated design of fault detection and FTC. But such a task is significantly beyond the scope of this paper.

In the simulation, the initial conditions are $\mathbf{q}(0)=$ $\left[\begin{array}{llll}1 & 0 & 0 & 0\end{array}\right]$ and $\mathbf{q}_{e}(0)=\mathbf{0}$ and the variance of the disturbance noise $d(t)$ is $\sigma_{d}^{2}=0.01$. The single fault $f_{2}(t)$ is considered in both cases, while this fault is modeled as the step function with amplitude equal to one and continuing from the starting time instant $t=50 \mathrm{~s}$.

In Figures 1 and 2 are shown the singular values plot (a) and the fault detection filter response (b), both for the systems under state control in autonomous regime. The value $s_{o}=5$ was chosen in order to not decelerate the observer dynamics conditioned by the stable eigenvalues of $\mathbf{A}_{\text {o22 }}$.

Example 2. The unstable system is represented by the chemical reactor model [36] in the form (1). The system model matrices are given as follows:

$$
\begin{aligned}
& \mathbf{A}=\left[\begin{array}{cccc}
1.380 & -2.080 & 6.715 & -5.676 \\
-0.581 & -4.290 & 0.000 & 0.675 \\
10.672 & 4.273 & -6.654 & 5.893 \\
0.482 & 4.273 & 1.343 & -2.104
\end{array}\right] \text {, } \\
& \mathbf{B}=\left[\begin{array}{ccc}
0.000 & 0.000 & 0.000 \\
5.679 & -1.000 & 0.000 \\
1.136 & -3.146 & 1.324 \\
1.136 & 0.000 & 3.496
\end{array}\right], \\
& \mathbf{F}=\left[\begin{array}{ccc}
0.000 & 0.000 & 0.000 \\
5.679 & -1.000 & 0.000 \\
1.136 & -3.146 & 1.324 \\
1.136 & 0.000 & 3.496
\end{array}\right], \\
& \mathbf{E}=\left[\begin{array}{l}
1.400 \\
1.504 \\
2.233 \\
0.610
\end{array}\right] \text {, } \\
& \mathbf{C}=\left[\begin{array}{llll}
4 & 0 & 1 & 0 \\
0 & 0 & 0 & 1 \\
0 & 1 & 0 & 0
\end{array}\right] \text {, }
\end{aligned}
$$

with the corresponding parameters, as defined in (9) and (10),

$$
\begin{array}{r}
\mathbf{V}=\mathbf{C F}=\left[\begin{array}{ccc}
1.136 & -3.146 & 1.324 \\
1.136 & 0.000 & 3.496 \\
5.679 & -1.000 & 0.000
\end{array}\right], \\
\operatorname{det}(\mathbf{V}) \neq 0,
\end{array}
$$




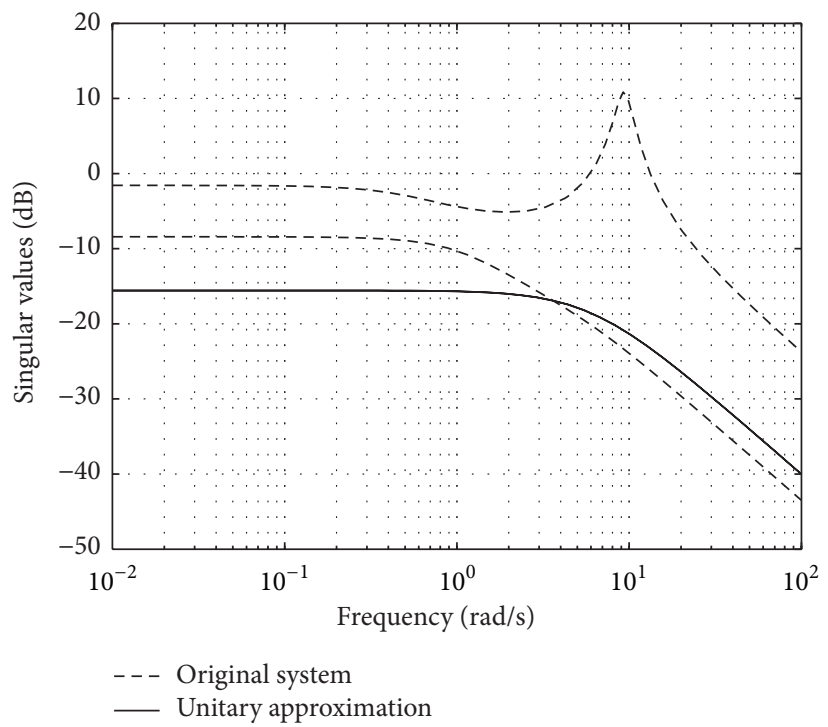

(a)

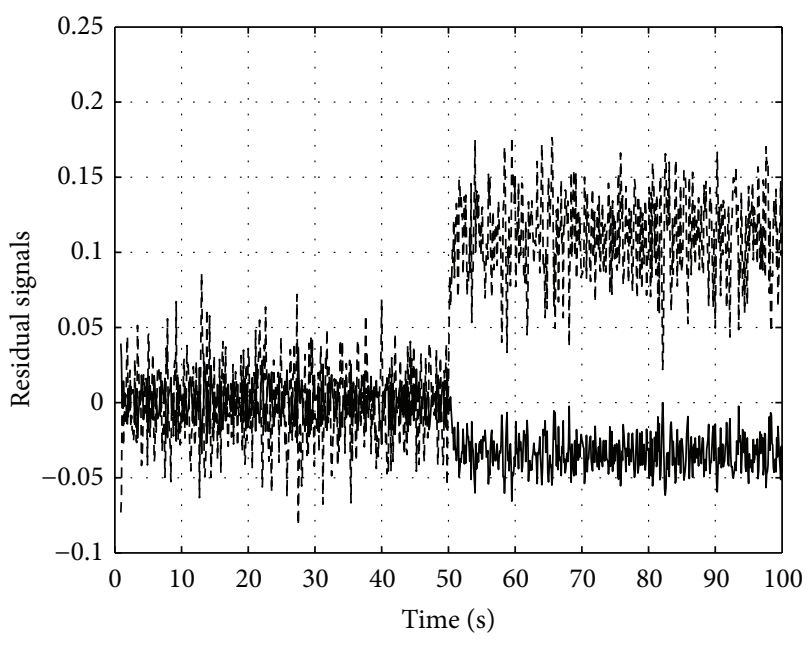

$-r_{1}$

(b)

Figure 1: (a) Singular values plot. (b) Residuals.

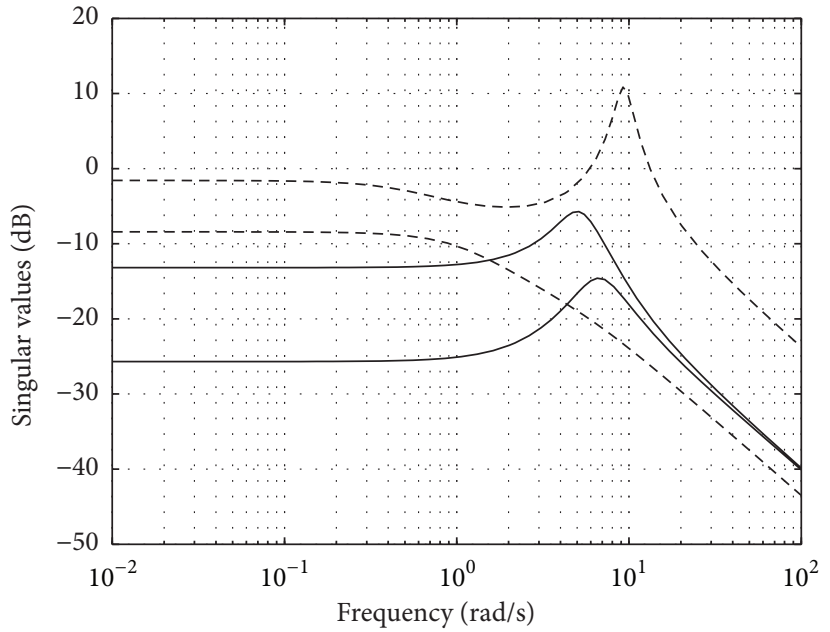

-- - Original system

_ Unitary approximation

(a)

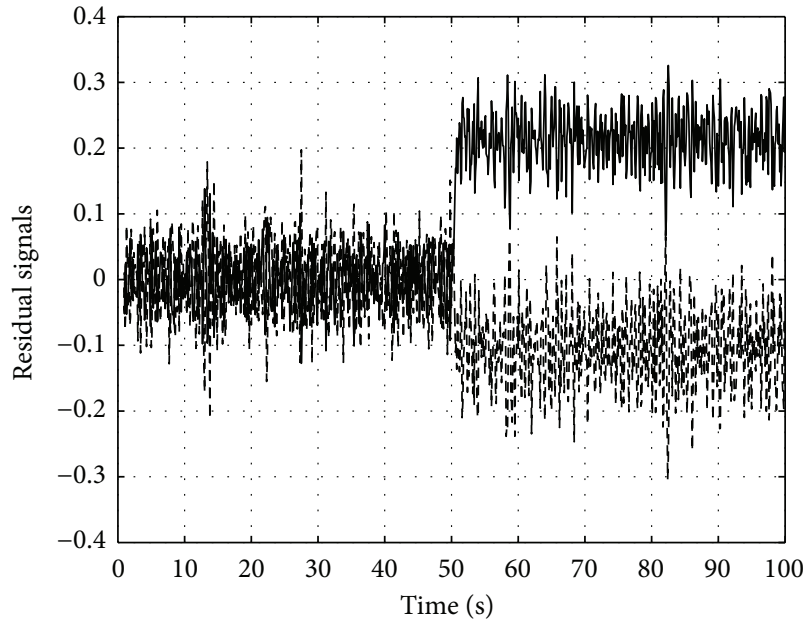

$-r_{1}$

(b)

Figure 2: (a) Singular values plot. (b) Residuals.

$$
\begin{aligned}
\mathbf{V}^{-1} \mathbf{C} & =\left[\begin{array}{cccc}
-0.2331 & 0.1833 & -0.0583 & 0.0221 \\
-1.3237 & 0.0411 & -0.3309 & 0.1253 \\
0.0757 & -0.0596 & 0.0189 & 0.2789
\end{array}\right], \\
\mathbf{F}^{\perp} & =\left[\begin{array}{llll}
-1.0000 & 0.0000 & 0.0000 & 0.0000
\end{array}\right], \\
\mathbf{T} & =\left[\begin{array}{cccc}
-0.2331 & 0.1833 & -0.0583 & 0.0221 \\
-1.3237 & 0.0411 & -0.3309 & 0.1253 \\
0.0757 & -0.0596 & 0.0189 & 0.2789 \\
-1.0000 & 0.0000 & 0.0000 & 0.0000
\end{array}\right] .
\end{aligned}
$$

Referring to (20), for the blocks of $\mathbf{A}^{\circ}$, the following terms are computed:

$$
\begin{aligned}
& \mathbf{A}_{o 11}=\left[\begin{array}{ccc}
-6.1156 & 4.1078 & -0.9991 \\
-11.1342 & 19.0383 & -8.0335 \\
8.6811 & -3.7656 & -1.2707
\end{array}\right], \\
& \mathbf{A}_{o 12}=\left[\begin{array}{c}
-3.8116 \\
-21.0651 \\
2.7614
\end{array}\right]
\end{aligned}
$$




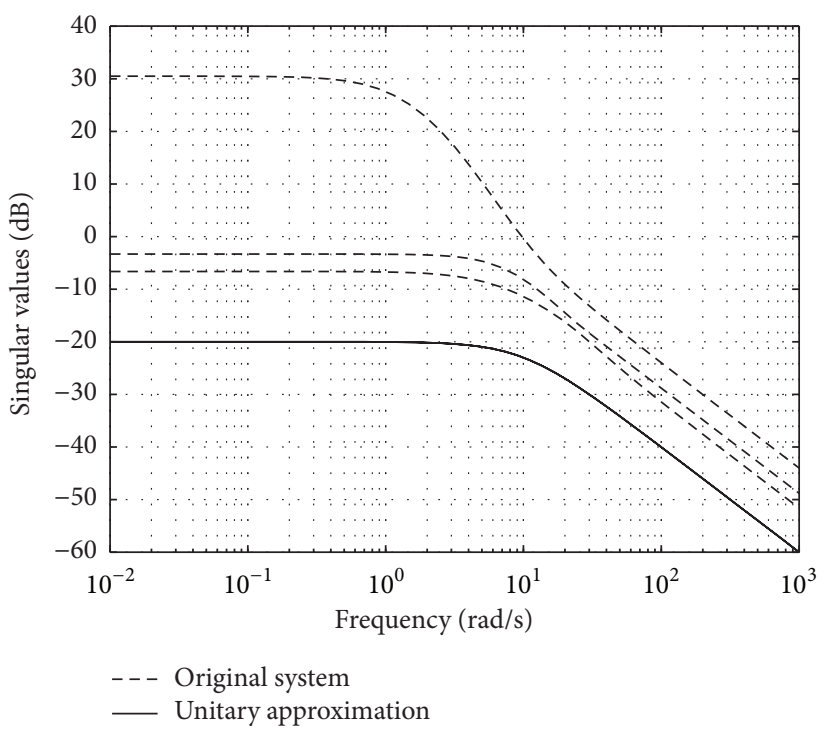

(a)

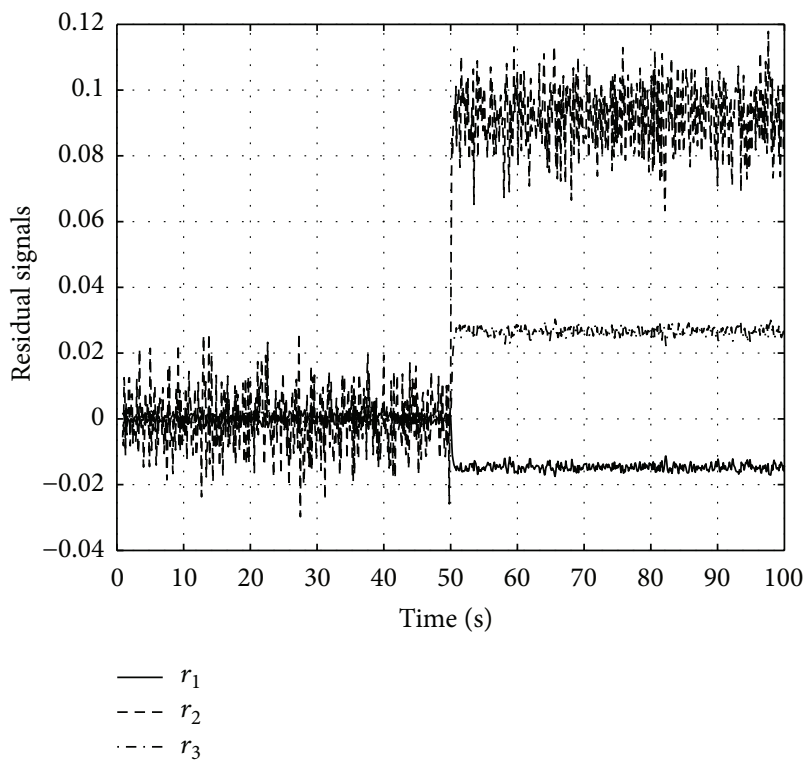

(b)

FIGURE 3: (a) Singular values plot. (b) Residuals.

$$
\begin{aligned}
& \mathbf{A}_{o 21}=\left[\begin{array}{lll}
-4.0432 & 19.2186 & -2.6660
\end{array}\right], \\
& \mathbf{A}_{\text {o22 }}=[-23.3200] \text {, }
\end{aligned}
$$

implying that the fault detection filter with a unitary transfer function can be designed. Choosing $s_{o}=9$, it is obtained using (21) and (66) that

$$
\begin{aligned}
\mathbf{L}^{o} & =\left[\begin{array}{ccc}
2.8844 & 4.1078 & -0.9991 \\
-11.1342 & 28.0383 & -8.0335 \\
8.6811 & -3.7656 & 7.7293 \\
-4.0432 & 19.2186 & -2.6660
\end{array}\right], \\
\mathbf{M} & =\left[\begin{array}{ccc}
6.1750 & -1.5760 & -0.2080 \\
0.0000 & 0.6750 & 4.7100 \\
2.3460 & 5.8930 & 4.2730 \\
1.3430 & 6.8960 & 4.2730
\end{array}\right], \\
\mathbf{N} & =\left[\begin{array}{ccc}
0 & 0 & 0 \\
0 & 0 & -1 \\
-1 & 0 & 0 \\
0 & -1 & 0
\end{array}\right], \\
\mathbf{J} & =\left[\begin{array}{lll}
6.1750 & -1.5760 & -0.2080 \\
0.0000 & 0.6750 & 5.7100 \\
3.3460 & 5.8930 & 4.2730 \\
1.3430 & 7.8960 & 4.2730
\end{array}\right],
\end{aligned}
$$

while the eigenvalues spectrum of $\mathbf{A}_{e}$ and the steady-state value of the fault detection filter transfer function matrix $\mathbf{G}_{f}(0)$ are

$$
\begin{aligned}
& \rho\left(\mathbf{A}_{e}\right)=\left\{\begin{array}{llll}
-23.32 & -10.00 & -10.00 & -10.00
\end{array}\right\}, \\
& \mathbf{G}_{f}(0)=-\mathbf{V}^{-1} \mathbf{C A}_{e}^{-1} \mathbf{F}=\left[\begin{array}{ccc}
0.1 & 0.0 & 0.0 \\
0.0 & 0.1 & 0.0 \\
0.0 & 0.0 & 0.1
\end{array}\right] \text {, }
\end{aligned}
$$

respectively. It is evident that for all diagonal elements of $\mathbf{G}_{f}(0)$ it yields the relation $\left(s_{o}+1\right)^{-1}=0.1$.

Also in this example the matrix $\mathbf{A}$ is not Hurwitz and the system is stabilized using the state feedback control law. Designing, for simplicity, with the prescribed set of closedloop system matrix eigenvalues $\rho(\mathbf{A}-\mathbf{B K})=\{-2,-3,-3,-4\}$, the gain matrix is computed as follows:

$$
\mathbf{K}=\left[\begin{array}{cccc}
-0.9686 & -0.2676 & -0.1543 & 0.1358 \\
-4.1485 & -0.6006 & 0.3189 & -0.9145 \\
0.0893 & 1.4926 & -0.1525 & 0.7086
\end{array}\right]
$$

In the simulation, the initial conditions are $\mathbf{q}(0)=$ $\left[\begin{array}{llll}0 & 1 & 0 & 0\end{array}\right]$ and $\mathbf{q}_{e}(0)=\mathbf{0}$ and the variance of the disturbance noise $d(t)$ is $\sigma_{d}^{2}=0.01$, while a single fault on the second actuator is considered. The fault is initialized at the time instant $t=50 \mathrm{~s}$ as an additive step function with the amplitude equal to one.

In Figure 3 are shown the singular values plot (a) and the fault detection filter response (b), both for the system under state control in autonomous regime. Since the stable 
eigenvalue of $\mathbf{A}_{o 22}$ determines the sufficiently fast estimator dynamics, the value $s_{o}=9$ is chosen only from the numerical point of view. Moreover, it is possible to see in Figure 3 the directional properties of the output signals of the fault detection filter.

\section{Concluding Remarks}

The approach of solving a unitary approximation of a square fault detection filter transfer function matrix is presented in the context of multiple singular values design, where the conditions for existence of a unitary construction are presented. If the design conditions are satisfied, by choosing one related singular value, the explicit relations for the filter gain matrix design are obtained, which gives a stable fault detection filter with a unitary transfer function to provide high residual signals sensitivity with respect to faults.

Otherwise, reflecting the emplacement of singular values in unitary construction principle and combining the resulting filter gain matrix but for a structure with unstable set of observer system matrix eigenvalues, an associated structure of linear matrix inequalities, as well as one matrix equality together with built-in state and output variable constraints, is outlined to compute an additive stabilizable gain matrix and, in consequence, to design the fault detection filter but only with a Hurwitz transfer function. Formulated in sense of the second Lyapunov method, stability conditions guaranteeing the asymptotic convergence of fault detection filter state are derived for continuous-time linear systems. The numerical simulation results show very good approximation performances.

Although the results represent an improvement on solutions, some conservatism may exist since a common matrix variable is required to satisfy the LMI with a quadratic constraint, but only for systems which do not satisfy the matching condition, that is, when the fault detection filter with a Hurwitz transfer function matrix has to be designed. Although the design conditions are not formulated in terms of robust stability, under nominal occasions, the robustness is flattened to comparable design methods [38-40]. The robustness still remains an open and challenging problem.

\section{Competing Interests}

The authors declare that they have no competing interests.

\section{Acknowledgments}

The work presented in this paper was supported by VEGA, the Grant Agency of the Ministry of Education, and the Academy of Science of Slovak Republic, under Grant no. $1 / 0348 / 14$. This support is gratefully acknowledged.

\section{References}

[1] M. Blanke, M. Kinnaert, J. Lunze, and M. Staroswiecki, Diagnosis and Fault-Tolerant Control, Springer, Berlin, Germany, 2006.
[2] M. S. Mahmoud and Y. Xia, Analysis and Synthesis of FaultTolerant Control Systems, John Wiley \& Sons, Chichester, UK, 2014.

[3] H. Noura, D. Theilliol, J. C. Ponsart, and A. Chamseddine, Faulttolerant Control Systems: Design and Practical Applications, Springer, London, UK, 2009.

[4] K. Zhang, B. Jiang, and P. Shi, Observer-Based Fault Estimation and Accomodation for Dynamic Systems, Springer, Berlin, Germany, 2013.

[5] S. Ding, Model-Based Fault Diagnosis Techniques. Design Schemes, Algorithms, and Tools, Springer, Berlin, Germany, 2008.

[6] A. Zolghadri, D. Henry, J. Cieslak, D. Efimov, and P. Goupil, Fault Diagnosis and Fault-Tolerant Control and Guidance for Aerospace Vehicles. From Theory to Application, Springer, London, UK, 2014.

[7] J. Chen and R. J. Patton, Robust Model-Based Fault Diagnosis for Dynamic System, Kluwer Academic, Norwell, Mass, USA, 1999.

[8] M. Hou and R. J. Patton, "An LMI approach to $H_{-} / H_{\infty}$ fault detection observers," in Proceedings of the UKACC International Conference on Control (CONTROL '96), pp. 305-310, Exeter, UK, September 1996.

[9] S. X. Ding, T. Jeinsch, P. M. Frank, and E. L. Ding, "A unified approach to the optimization of fault detection systems," International Journal of Adaptive Control and Signal Processing, vol. 14, no. 7, pp. 725-745, 2000.

[10] N. Liu and K. Zhou, "Optimal robust fault detection for linear discrete time systems," Journal of Control Science and Engineering, vol. 2008, Article ID 829459, 16 pages, 2008.

[11] W. Ding, Z. Mao, B. Jiang, and W. Chen, "Fault detection for a class of nonlinear networked control systems with Markov transfer delays and stochastic packet drops," Circuits, Systems, and Signal Processing, vol. 34, no. 4, pp. 1211-1231, 2015.

[12] Y. Zhang, L. Wu, J. Li, and X. Chen, "LMI approach to mixed $H_{-} / H_{\infty}$ fault detection observer design," Transactions of Tianjin University, vol. 18, no. 5, pp. 343-349, 2012.

[13] Z. Zhao, W.-F. Xie, H. Hong, and Y. Zhang, "Unitary system I. Constructing a unitary fault detection observer," in Proceedings of the 18th IFAC World Congress, pp. 7725-7730, Milano, Italy, 2011.

[14] Z. Zhao, W.-F. Xie, H. Hong, and Y. Zhang, "Unitary system II. Application to $\mathrm{H} 1 / \mathrm{H}$ optimization of strictly-proper systems," in Proceedings of the 18th IFAC World Congress, pp. 7731-7736, Milano, Italy, 2011.

[15] D. Krokavec and A. Filasová, "Construction process of unitary systems using LMI based approach," IFAC-PapersOnLine, vol. 48, no. 14, pp. 174-179, 2015.

[16] D. Krokavec, A. Filasová, and P. Liščinský, "On fault detection filters design with unitary transfer function matrices," Journal of Physics: Conference Series, vol. 659, no. 1, Article ID 012036, 2015.

[17] F. N. Chowdhury and W. Chen, "A modified approach to observer-based fault detection," in Proceedings of the IEEE Multi-Conference on Systems and Control, 16th IEEE International Conference on Control Applications, pp. 946-951, Singapore, 2007.

[18] T. Y. Azizov and I. S. Iokhvidov, Linear Operators in Spaces with an Indefinite Metric, John Wiley \& Sons, Chichester, UK, 1989.

[19] M. H. Yang, "The state space of a canonical linear system," Journal of the Korean Mathematical Society, vol. 32, no. 3, pp. 447-459, 1995. 
[20] L. Debnath and D. Bhatta, Integral Transforms and Their Applications, Chapman \& Hall, Boca Raton, Fla, USA, 2007.

[21] S. Toffner-Clausen, System Identification and Robust Control. A Case Study Approach, Springer, London, UK, 1996.

[22] B. A. Francis, Ed., A Course in $H_{\infty}$ Control Theory, vol. 88 of Lecture Notes in Control and Information Sciences, Springer, Berlin, Germany, 1987.

[23] D. Boyd and V. Balakrishnan, "A regularity result for the singular values of a transfer matrix and a qudratically convergent algorithm for computing its L1-norm," Systems \& Control Letters, vol. 15, no. 1, pp. 1-7, 1990.

[24] X. Li and H. H. T. Liu, "Minimum system sensitivity study of linear discrete time systems for fault detection," Mathematical Problems in Engineering, vol. 2013, Article ID 276987, 13 pages, 2013.

[25] Y. Li, T. Zhang, X. Liu, and X. Jiang, "Study on $H_{-}$index of stochastic linear continuous-time systems," Mathematical Problems in Engineering, vol. 2015, Article ID 837053, 10 pages, 2015.

[26] J. C. Doyle, B. A. Francis, and A. R. Tannenbaum, Feedback Control Theory, Maxwell Macmillan International, Singapore, 1992.

[27] M. Green and D. J. N. Limebeer, Linear Robust Control, Prentice Hall, Englewood Cliffs, NJ, USA, 1995.

[28] O. N. Gasparyan, Linear and Nonlinear Multivariable Feedback Control. A Classical Approach, John Wiley \& Sons, Chichester, UK, 2008.

[29] D. S. Bernstein, Matrix Mathematics: Theory, Facts, and Formulas, Princeton University Press, Princeton, NJ, USA, 2009.

[30] W. A. Adkins and S. H. Weintraub, Algebra-An Approach via Module Theory, Springer, Berlin, Germany, 1992.

[31] F. W. Fairman, Linear Control Theory: The State Space Approach, John Wiley \& Sons, Chichester, UK, 1998.

[32] J. W. Miles, Integral Transforms in Applied Mathematics, Cambridge University Press, New York, NY, USA, 1971.

[33] H. K. Khalil, Nonlinear Systems, Prentice Hall, Upper Saddle River, NJ, USA, 2002.

[34] D. G. Schultz and J. L. Melsa, State Functions and Linear Control Systems, McGraw-Hill, New York, NY, USA, 1967.

[35] W. M. Haddad and V. Chellaboina, Nonlinear Dynamical Systems and Control. A Lyapunov-Based Approach, Princeton University Press, Princeton, NJ, USA, 2008.

[36] J. Kautsky, N. K. Nichols, and P. Van Dooren, "Robust pole assignment in linear state feedback," International Journal of Control, vol. 41, no. 5, pp. 1129-1155, 1985.

[37] D. Krokavec and A. Filasová, "LMI constraints on system eigenvalues placement in dynamic output control design," in Proceedings of the IEEE Conference on Control Applications (CCA '15), pp. 1749-1754, Sydney, Australia, September 2015.

[38] I. M. Jaimoukha, Z. Li, and V. Papakos, "A matrix factorization solution to the $H_{-} / H_{\infty}$ fault detection problem," Automatica, vol. 42, no. 11, pp. 1907-1912, 2006.

[39] A. Saberi, A. A. Stoorvogel, and P. Sannuti, Filtering Theory. With Applications to Fault Detection, Isolation, and Estimation, Birkhaüser, Boston, Mass, USA, 2007.

[40] X. Li, H. H. T. Liu, and B. Jiang, "Fault detection filter design with optimization and partial decoupling," IEEE Transactions on Automatic Control, vol. 60, no. 7, pp. 1951-1956, 2015. 


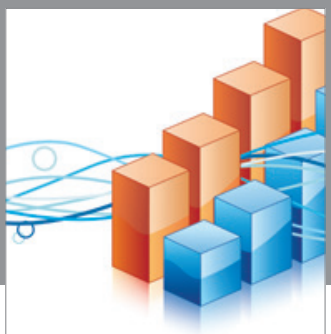

Advances in

Operations Research

vatem alat4

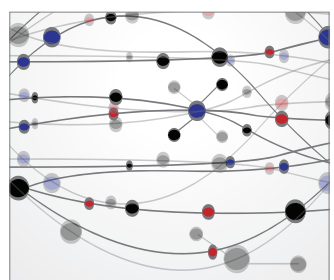

\section{The Scientific} World Journal
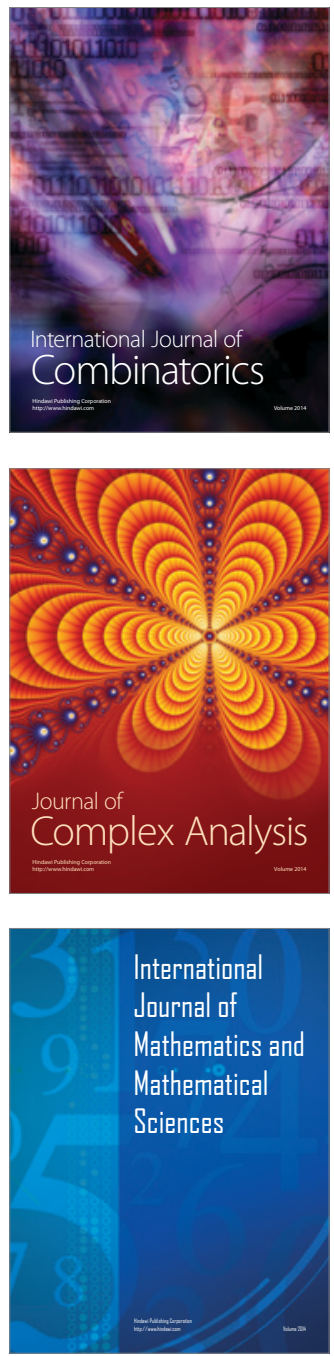
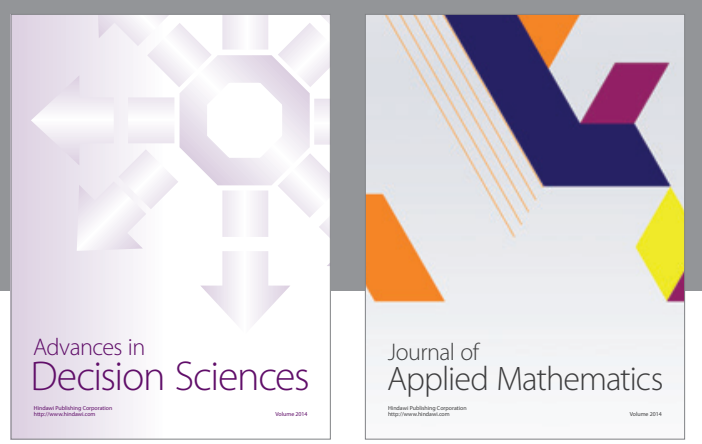

Algebra

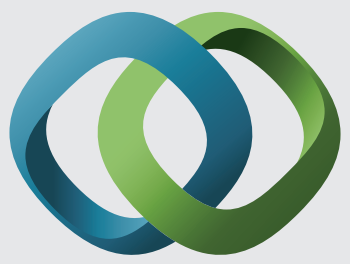

\section{Hindawi}

Submit your manuscripts at

http://www.hindawi.com
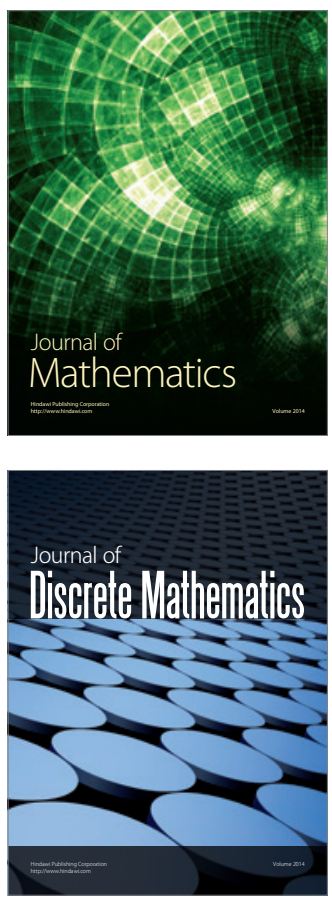

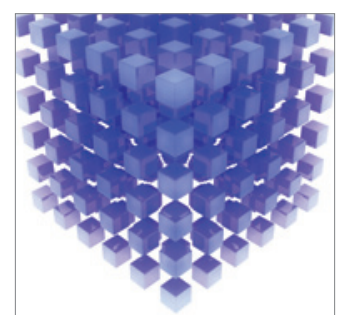

Mathematical Problems in Engineering
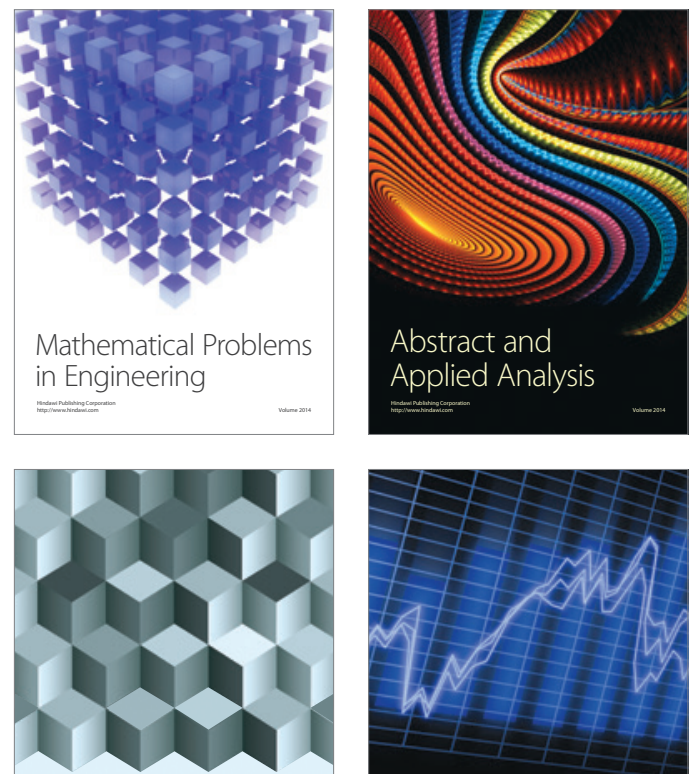

Journal of

Function Spaces

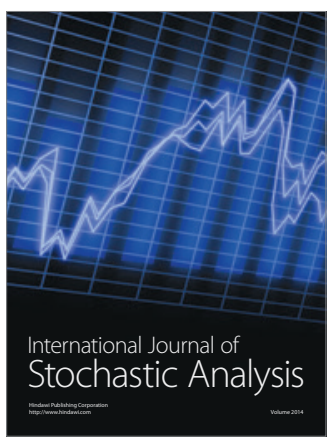

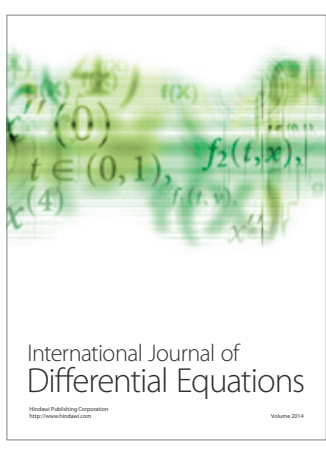
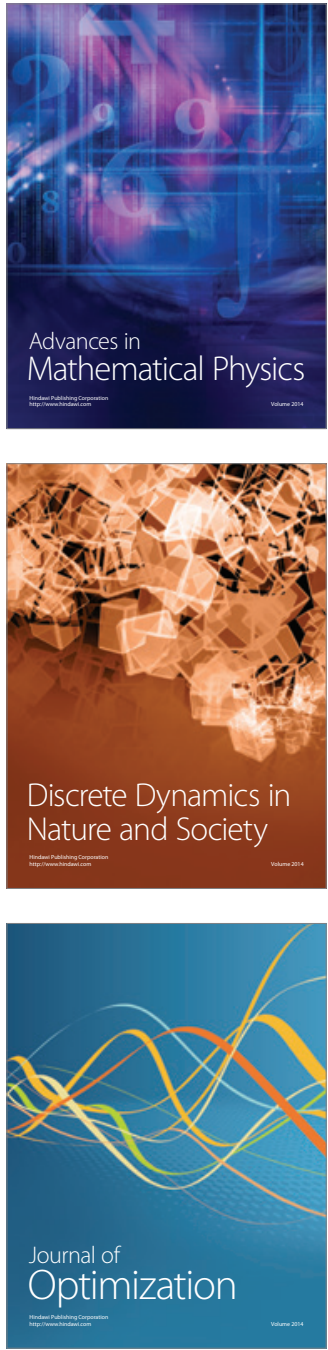